\title{
Kinematic Structure of Mesovortices in the Eyewall of Hurricane Ike (2008) Derived from Ground-Based Dual-Doppler Analysis ${ }^{\mathscr{O}}$
}

\author{
STEPHANIE M. WINGO a AND KEVIN R. KNUPP \\ Severe Weather Institute and Radar and Lightning Laboratories, University of Alabama \\ in Huntsville, Huntsville, Alabama
}

(Manuscript received 8 March 2016, in final form 3 August 2016)

\begin{abstract}
Previous work has shown that vorticity mixing in the tropical cyclone (TC) inner core can promote mesovortex (MV) formation and impact storm intensity. Observations of MVs have largely been serendipitous but are necessary to improve understanding of these features and their role in TC dynamics. This study presents nearly $10 \mathrm{~h}$ of ground-based dual-Doppler analysis of MVs in the eyewall of Hurricane Ike (2008) near and during landfall. Derived 3D winds, vertical vorticity, horizontal divergence, and perturbation pressures are analyzed. Results indicate persistent kinematic field arrangements and evolving vertical structures. Perturbation pressure retrievals suggest local pressure minima associated with the MVs. Preferential updraft locations appear to transition cyclonically about the local vorticity maximum as the MVs progress around the eye. Based on published observational datasets, the dual-Doppler updraft magnitudes in Ike's MVs are within the top 5\%-10\% of TC vertical velocities. The MVs are marked by peak vorticity in the lowest $2 \mathrm{~km}$ and contain vertically coherent vorticity structures extending to $8 \mathrm{~km}$ AGL. After prolonged land interaction, the MV structures deteriorate. First, the vertical extent of localized vorticity diminishes, followed by a deterioration in the prelandfall characteristic kinematic arrangements. This supports the notion that the replenishment of a high vorticity annulus contributes to MV production and maintenance, and when the elevated vorticity aloft is not maintained, MV kinematic patterns become less consistent. It is unclear whether the decay of the vertically coherent vorticity structures occurs in response to land interaction, TC inner core processes, or some combination of both.
\end{abstract}

\section{Introduction}

Early observations of tropical cyclones (TCs) revealed the occurrence of coherent, mesoscale structures, including mesovortices (MVs), embedded in the eyewall (Lewis and Hawkins 1982). Several cases of TC eyewall MVs have been examined in detail. Marks and Houze (1984) found evidence for an MV in the eyewall of Hurricane Debby (1982). An MV encountered by a research aircraft in Hurricane Hugo (1989) provided the first in situ MV observations, which suggested the feature may have aided

Supplemental information related to this paper is available at the Journals Online website: http://dx.doi.org/10.1175/ MWR-D-16-0085.s1.

${ }^{a}$ Current affiliation: Science Systems and Applications, Inc., NASA Wallops Flight Facility, Wallops Island, Virginia.

Corresponding author address: Stephanie M. Wingo, Science Systems and Applications, Inc., NASA Wallops Flight Facility, Code 612, Building N-159, Room E-213, Wallops Island, VA 23337.

E-mail: stephanie.m.wingo@nasa.gov intensification via an eye-eyewall mixing process (Marks et al. 2008). With flight-level and airborne radar data, Reasor et al. (2000) showed that Hurricane Olivia (1994) compared well to simulations of eyewall breakdown. Kossin and Schubert (2004) noted a remarkable similarity between Hurricane Isabel (2003) and numerical simulations (Schubert et al. 1999; Kossin and Schubert 2001). Rapid intensification of Hurricane Guillermo (1997) was observed in association with convective bursts related to low-wavenumber vorticity asymmetries (Reasor et al. 2009). For the sheared case of Tropical Storm Gabrielle (2001), Molinari et al. (2006) described the process of an MV becoming the primary TC center. Hendricks et al. (2012) showed the asymmetric inner core evolution observed by a single ground-based radar in Hurricane Dolly (2008) during rapid intensification about $6 \mathrm{~h}$ prior to landfall. The TC inner core MVs have also been viewed with synthetic aperture radar from space ( $\mathrm{Li}$ et al. 2013). Regardless of the platform, however, observations of these features have been serendipitous at best, but represent a key avenue to understanding the dynamics 
governing MV formation, evolution, and storm intensity impacts. In this paper, we document the kinematic structure of MVs observed in Hurricane Ike (2008) with ground-based dual-Doppler analyses.

The TC eyewall can be simplified as an annulus of elevated potential vorticity (PV), generated in large part by diabatic heating. Several idealized simulation studies have shown that this ring supports the development of counterpropagating vortex Rossby waves on the opposite-signed PV gradients. The annulus becomes barotropically unstable as these waves lock in phase and grow exponentially, resulting in the breakdown of the ring into MVs and the development of polygonal eyewalls, MV mergers, and intensity changes for the parent vortex (Schubert et al. 1999; Kossin and Schubert 2001; Terwey and Montgomery 2002; Kossin and Schubert 2004; Hendricks et al. 2009; Martinez et al. 2010). Analyzed flight-level data revealed distinct regimes in eyewall kinematic and thermodynamic profiles indicative of vorticity mixing that correspond well to these simulations (Kossin and Eastin 2001).

Persing and Montgomery (2003) proposed a "turboboost" mechanism by which a TC can intensify beyond predicted limits. Results for the Isabel case generally supported this notion (Montgomery et al. 2006; Aberson et al. 2006; Bell and Montgomery 2008). Mixing high entropy eye air into the eyewall allows the storm to tap an additional heat source and increase local instability (Eastin et al. 2005). Braun et al.'s (2006) high-resolution, moist simulation of Hurricane Bonnie (1998) showed that MV features can interact with shear-induced low level flow, initiating updrafts and enhancing eyewall convection. Trajectory analysis for Bonnie by Cram et al. (2007) showed transport of positively buoyant, low-level eye air into the eyewall, supporting the turboboost mechanism. While these results showed MV episodes can support significant intensification, Rozoff et al. (2009) discussed a dual nature of vorticity mixing in which the breakdown of the eyewall initially produces decreased maximum winds, but MV-facilitated eye-eyewall interactions eventually result in a more intense TC and deep convection in the eyewall continuously reinvigorates the vorticity annulus.

Hendricks and Schubert (2010) showed in a 3D, dry adiabatic simulation that PV mixing during MV episodes occurs mainly at low levels. Hendricks et al. (2012) presented observations supporting that result, and a simulation indicating barotropic instability as the "most plausible" driver of such asymmetries. Using a shallowwater model with parameterized heating, Hendricks et al. (2014) described a diabatic heating-induced divergent circulation that can advect PV, producing a thinner annulus that is more susceptible to barotropic instability and breakdown. Including diabatic heating (proportional to the relative vorticity) resulted in a final vortex with stronger peak winds and lower central pressure.

Wu et al.'s (2009) realistic simulation results reiterated the importance of diabatic heating in sustaining the PV annulus and showed that interactions between PV and friction can help maintain a narrow ring structure. Naylor and Schecter (2014) compared dry and full-physics simulations in a cloud resolving model and found that larger surface drag resulted in a more resilient annulus structure and, while moist convection played a key role in replenishing the ring, it had little effect on the initial development of asymmetries during annulus breakdown. In a realistic modeling study of an MV episode during the rapid intensification of Hurricane Wilma (2005), Menelaou et al. (2013) found evidence of vortex Rossby waves acting to decelerate (accelerate) the flow at (within and outside of) the radius of maximum winds and concluded that barotropic instability was also the most likely cause of the inner core asymmetries. Using 3D full-physics WRF simulations of mature TC-like vortices, Wu et al. (2016) showed that with diabatic heating the eyewall vorticity ring can maintain itself via a feedback process incorporating the secondary circulation.

We should further consider the threat MVs can pose to coastal populations. Willoughby and Black (1996) noted how MVs in Hurricane Andrew (1992) locally increased devastating impacts, and Montgomery et al. (2002) showed in the laboratory that local MV horizontal winds can be up to $50 \%$ greater than those of the main circulation. In the case of Tropical Storm Gabrielle (2001), reformation of the storm center on an MV resulted in both an intensity increase and an earlier landfall (Molinari et al. 2006; Nguyen and Molinari 2015). Impacts on intensity change and increases in local destructive potential at landfall warrant detailed analysis of TC inner core MV observations when possible.

One may consult Berg (2009) and Brown et al. (2010) for complete accounts of Ike's development, track, and impacts. Here, we examine observations collected during Ike's U.S. landfall in southeast Texas. Beginning on 12 September 2008, Ike experienced about $5-8 \mathrm{~m} \mathrm{~s}^{-1}$ of north-northeasterly deep-layer $(850-200 \mathrm{hPa})$ wind shear as it approached the coast (see shear vector direction shown in Fig. 1d). This moderate shear persisted through landfall. In the hours prior to landfall, an eye about $20 \mathrm{~km}$ across formed and winds increased to about $48 \mathrm{~m} \mathrm{~s}^{-1}$. Ike's intensity maintained near $43 \mathrm{~m} \mathrm{~s}^{-1}$ until about half the eye crossed the coastline. The hurricane's forward speed accelerated from about $5 \mathrm{~m} \mathrm{~s}^{-1}$ at about 0000 UTC (all times in UTC on 13 September) as it approached the coast to about $8 \mathrm{~m} \mathrm{~s}^{-1}$ near 1500 UTC and continued to increase as Ike moved inland, as shown in Fig. 2. Ike made 
landfall in the United States along the northern edge of Galveston Island at about 0700 UTC. Not unlike the Dolly case about two months earlier (Hendricks et al. 2012), Ike's eyewall exhibited polygonal shapes and possible MV features before and during landfall. Figure 1 provides radar reflectivity images at hourly intervals showing the progression of Ike during 00301130 UTC (more detail is evident in the animation of raw KHGX reflectivity and velocity for the period 0000-1300 UTC, included in the online supplemental material). The evolution of the inner core during this time was observed by the Houston-Galveston NEXRAD WSR-88D (KHGX) and the University of Alabama in Huntsville (UAH) Mobile Alabama X-band (MAX) polarimetric Doppler radar.

The purpose of this paper is to document the kinematic structure of Ike's eyewall MVs from the perspective of ground-based dual-Doppler analysis. Data and analysis methods are described in the next section. Section 3 provides a brief overview of the eyewall evolution near landfall. Results for kinematic fields derived from dual-Doppler analyses and discussions are presented in sections 4 and 5, respectively, and the final section summarizes the conclusions.

\section{Data and methodology}

Observations of MVs from airborne and ground-based radars have been reported over at least the last three decades. However, to the authors' knowledge this is the first work examining these features with dual-Doppler analysis from ground-based radars. Level II data from KHGX and observations collected by the MAX radar are used [MAX specifications are included in Asefi-Najafabady et al. (2010)]. KHGX, which had been upgraded to superresolution (NEXRAD build 10), operated in multiple volume coverage patterns (VCPs) during the Ike event (211, 121, and 212), though for all times in this study KHGX executed VCP 212. MAX operated at the Anahuac Airport, on the northeast side of Galveston Bay, forming a $53-\mathrm{km}$ baseline to the northeast of KHGX (Fig. 3). MAX performed volume scans ranging in elevation as shown in Table 1. From about 0000 to 0400 UTC, the MAX1 and MAX2 scans were executed. After this, MAX1 and MAX3 scans were alternated. Because of more complete vertical coverage, only MAX2 and MAX3 scans are used herein for analysis. We note that a wedge at $330^{\circ}-10^{\circ}$ azimuth was blocked below $10^{\circ}$ elevation to accommodate the MAX radar truck cab.

Data from both radars were edited using NCAR's Solo-II software (primarily to correct aliased velocities) and gridded to a $160 \mathrm{~km} \times 160 \mathrm{~km} \times 10 \mathrm{~km}$ Cartesian grid with a Cressman weighting function and radius of influence of $1.0 \mathrm{~km}$ in all directions using the REORDER software package. Procedures for synthesizing velocity observations from multiple Doppler radars were developed by Armijo (1969), Miller and Strauch (1974), Ray et al. (1980), Testud and Chong (1983), Chong et al. (1983), Chong and Testud (1983), and others. For this study, synthesis of Doppler velocities was done in CEDRIC (Mohr et al. 1986). KHGX and MAX radar volumes completed generally within $2 \mathrm{~min}$ (or less) of each other were paired together for synthesis. Vertical velocities were obtained by downward integration of the mass continuity equation (in an effort to mitigate limitations of radar sampling that does not extend well into the boundary layer) and subtracting bulk estimates of particle fall speed based on the maximum reflectivity value for the grid box (i.e., $w=W-v_{t}$, where $w$ is vertical velocity, $W$ is total vertical motion, and $v_{t}$ is the particles' terminal fall speed). Initially, the analysis was performed for the $160 \mathrm{~km} \times 160 \mathrm{~km} \times 10 \mathrm{~km}$ domain $(1.0 \mathrm{~km} \times 1.0 \mathrm{~km} \times 0.5 \mathrm{~km}$ grid spacing $)$ using a uniform advection equal to Ike's forward speed, as interpolated from the best track record.

A second, smaller domain (with identical spacing as the large grid) was then placed over each feature of interest (see Fig. 3). Features of interest were principally identified as eyewall areas containing localized "kinks" or arcs in reflectivity, as this has been shown to be a prevalent trait among TC eyewall MVs (e.g., Kossin and Schubert 2001, 2004). In most instances, the features of interest contained locally concentrated low level $(1.5 \mathrm{~km}$, all heights given as AGL) vertical vorticity in the initial, larger domain results. The smaller grid was generally $30 \mathrm{~km} \times 30 \mathrm{~km} \times 10 \mathrm{~km}$, however a few features required a slightly larger horizontal extent (40 km at most, see Table 2). Horizontal winds derived in the larger grid analysis were averaged over the entire (3D) area of the smaller domain and used as the uniform advection for synthesis over only the smaller grid. These smaller grids were the primary analysis domains in this study. Horizontal wind divergence (hereafter referred to simply as divergence), vertical vorticity (hereafter, $\zeta$ ) and Ike-relative radial and tangential wind components were computed from the resulting $3 \mathrm{D}$ wind field. Features containing a coherent, localized $\zeta$ maximum in the primary, smaller domain analysis were deemed MVs or inland MVs (listed as "MV" and "iMV" in Table 2, respectively), depending on the location of Ike's center. If an examined feature lacked a distinct local peak of positive $\zeta$, it was deemed non-MV ("nMV" in Table 2). While we did not require a specific low-level $\zeta$ threshold for MV identification, the values of these local $\zeta$ peaks are on the order of about $4.5 \times 10^{-3} \mathrm{~s}^{-1}$. 

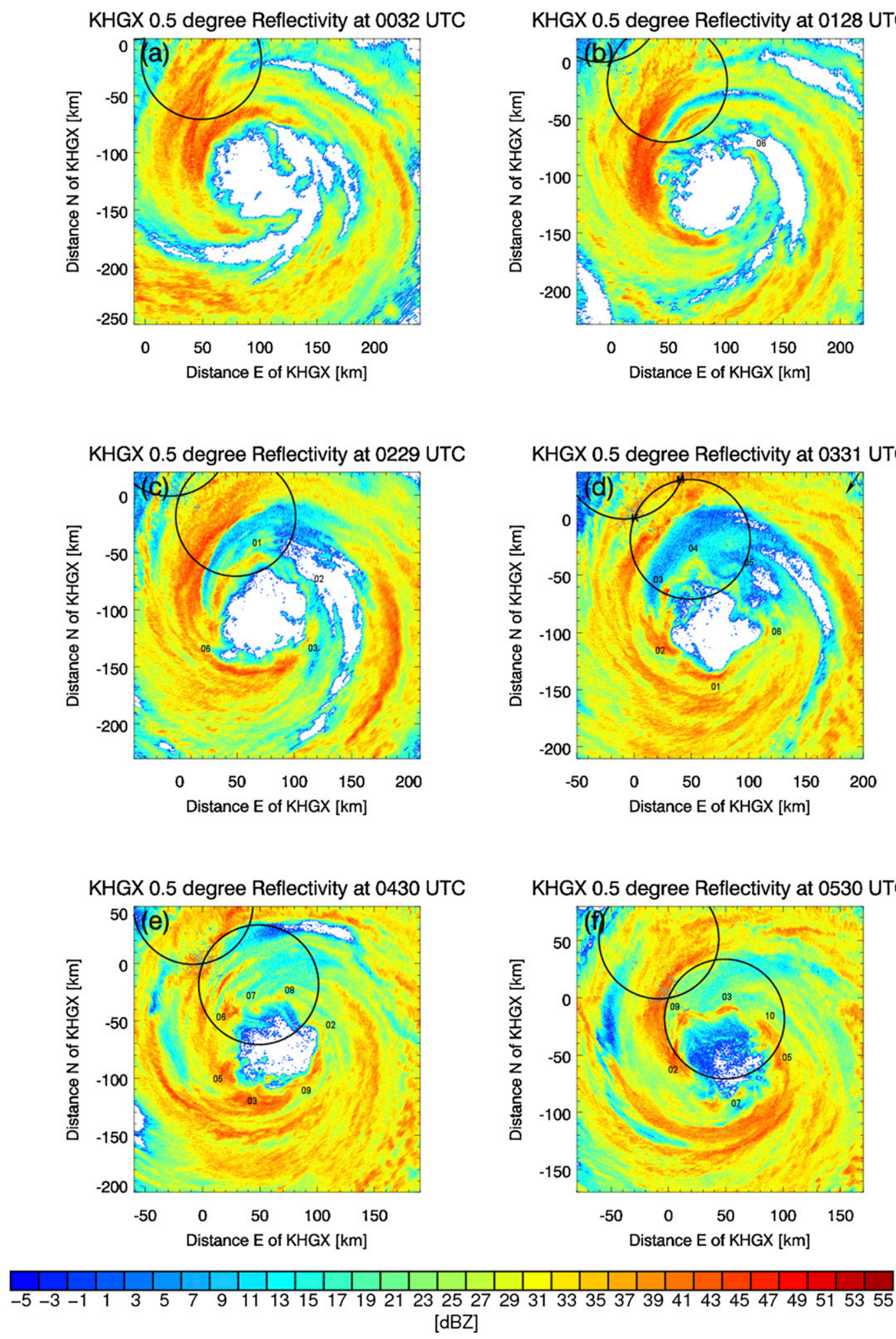

FIG. 1. Base elevation (0.5 ) PPI reflectivity from KHGX for 1-h intervals 0030-1130 UTC 13 Sep. In (d), the arrow at the top right indicates the direction of the deep layer shear vector, and the labels " $\mathrm{K}$ " and " $\mathrm{M}$ " mark locations of the KHGX and MAX radars, respectively. Circles indicate the KHGX-MAX dual-Doppler lobes, and numerals annotate the various MV features (numbers correspond to the list in Table 2). 

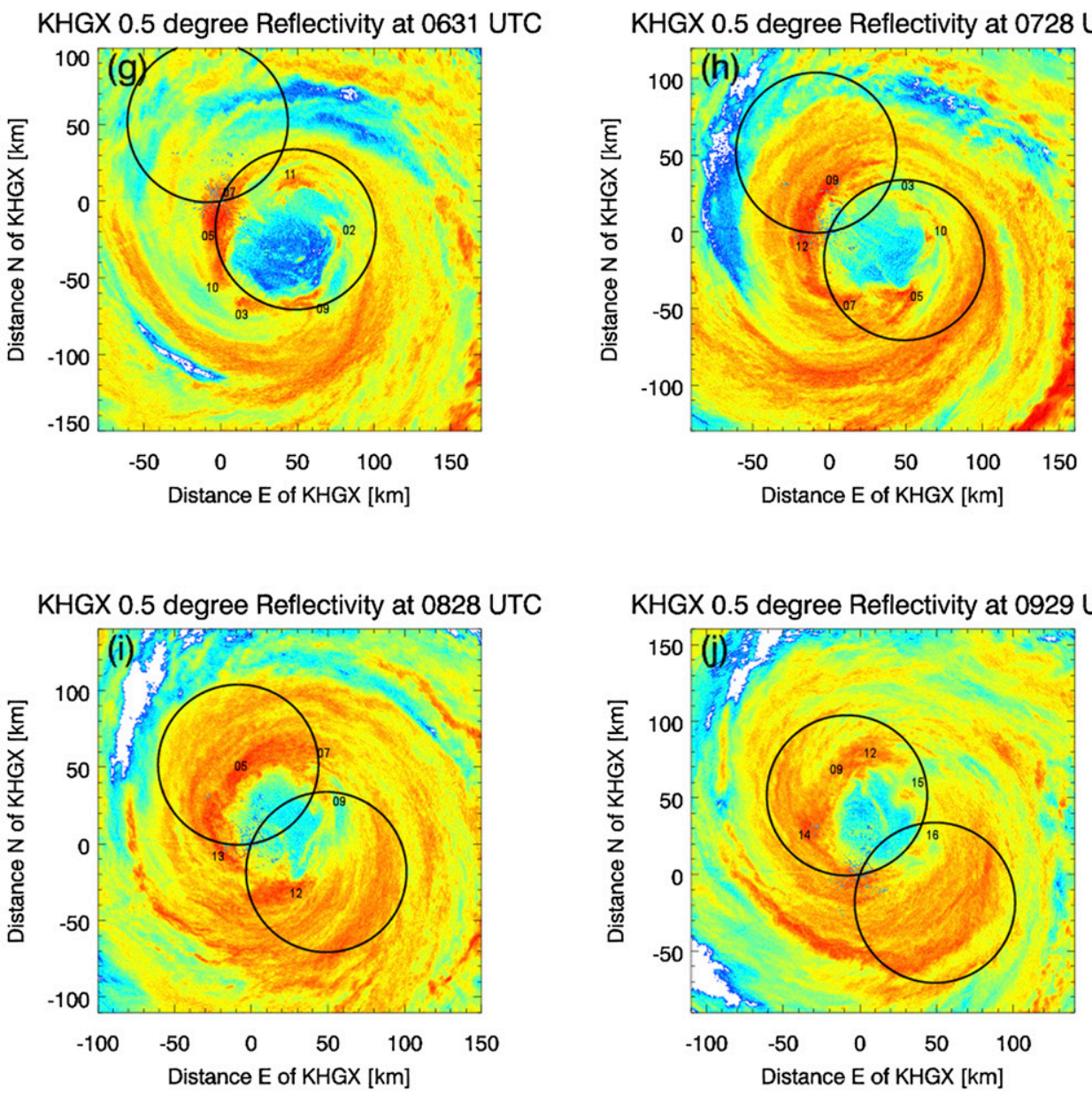

KHGX 0.5 degree Reflectivity at 1029 UTC
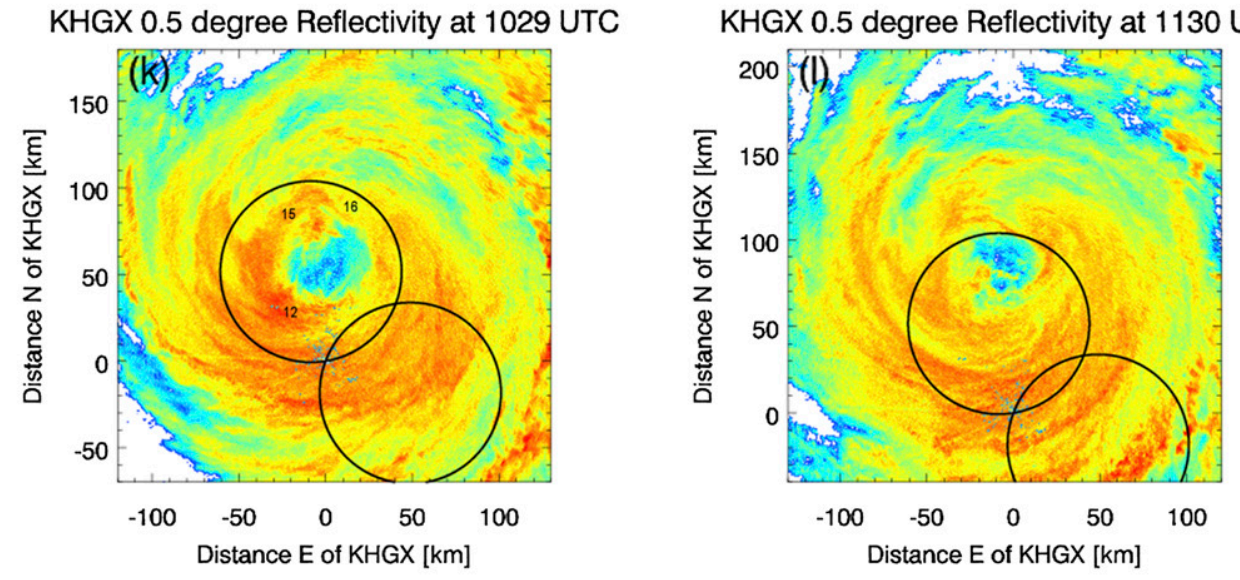

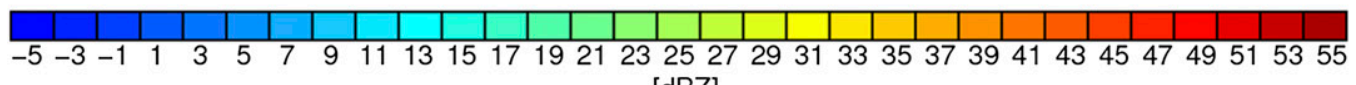
[dBZ]

FIG. 1. (Continued) 


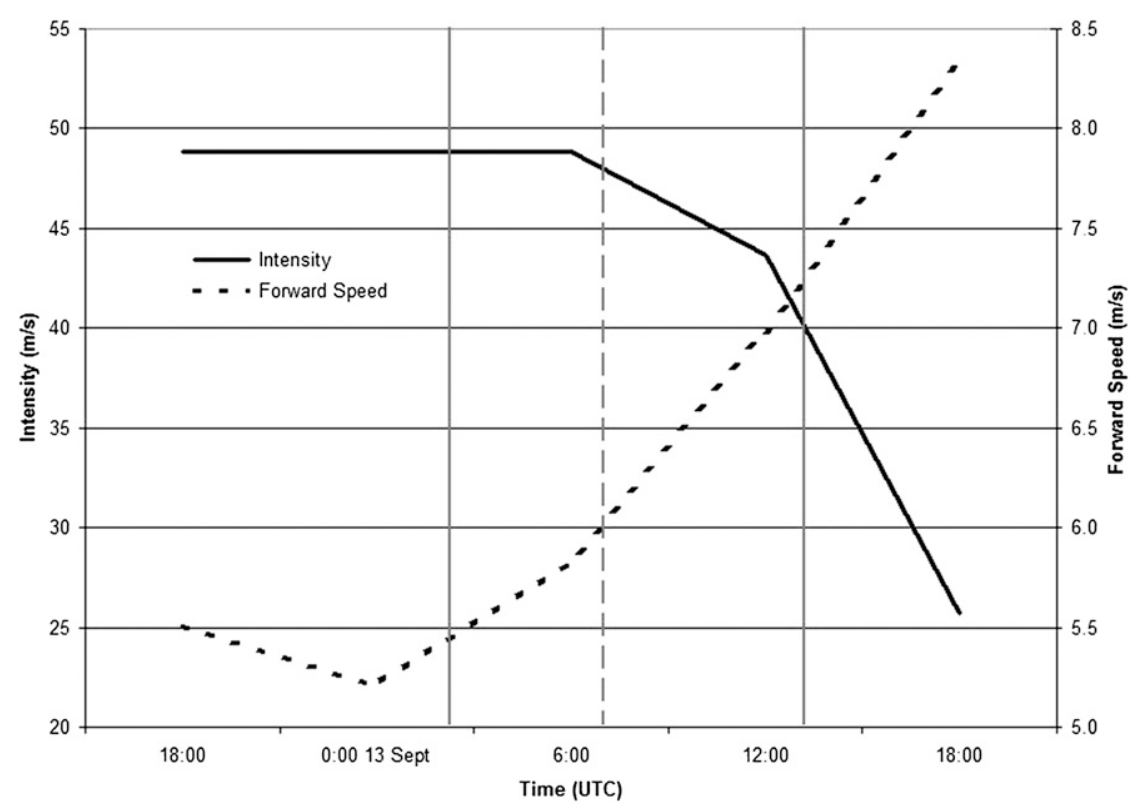

FIG. 2. Best track intensity ( $\mathrm{m} \mathrm{s}^{-1}$, left ordinate) and interpolated forward speed of Ike $\left(\mathrm{m} \mathrm{s}^{-1}\right.$, right ordinate). Solid gray vertical lines indicate the time interval when the eyewall was in the dualDoppler lobes, and the dashed gray vertical line denotes the time of landfall.

Dual-Doppler processing requires several assumptions that will affect the accuracy of our results. Storm evolution within the radar sample volume is likely over the time required for a full scan (about $5 \mathrm{~min}$ ). This steady-state assumption violation is compounded by inexact matching of the KHGX and MAX scan times, though as stated above, this was limited to about $2 \mathrm{~min}$ or less in an effort to minimize this problem. Systematic and random error sources, including turbulence in the radar sample volume and beam position inaccuracies due to deviations from the standard refraction model, can each contribute errors up to $1-2 \mathrm{~m} \mathrm{~s}^{-1}$ in the resulting horizontal wind components, and as with any dual-Doppler analysis, vertical velocities will be the least well-resolved wind component (Doviak et al. 1976; Doviak and Zrnić 1993). Additional issues for the $w$ retrievals are tied to the underdetermined nature of a dual-Doppler synthesis and accumulation of errors during the vertical continuity integration (Doviak et al. 1976; Clark et al. 1980; Matejka and Bartels 1998). Further complications arising in our application of the dual-Doppler technique for the Ike case include assumptions of uniform advection for both the large and small domain syntheses, assumption of a consistent TC center location with height (possibly contributing to aliasing of some of the tangential wind component into an incorrect radial component), and incomplete observations of the vertical profile of divergence. Base elevation scans from KHGX and MAX do not extend to the surface, leaving much of the boundary layer divergence unobserved, particularly at far ranges (at $60+\mathrm{km}$ from the radar, the lowest beam is at or above $1 \mathrm{~km} \mathrm{AGL)}$. Even with these concerns, the Ike case provides an opportunity for MV investigation, and qualitative aspects of our results represent an important documentation of TC eyewall MV structure and evolution just before and during landfall.

Once a 3D wind field is obtained, one can estimate perturbation pressure. The method used here is based on that of Gal-Chen (1978), and has been employed in several studies (e.g., Hane et al. 1981; Roux 1985; and recently, Murphy and Knupp 2013). This procedure begins with the anelastic form of the continuity and horizontal momentum equations. Solving that set for the pressure term leads to the following expression for the horizontal perturbation pressure field:

$$
\begin{aligned}
& \frac{\partial p^{\prime}}{\partial x}=F \equiv \frac{D u}{D x}+f_{x}+f r_{x}, \\
& \frac{\partial p^{\prime}}{\partial y}=G \equiv \frac{D v}{D y}+f_{y}+f r_{y},
\end{aligned}
$$

where $p^{\prime}$ is the perturbation pressure; $u$ and $v$ are zonal and meridional wind components, respectively; $f_{x}$ and $f_{y}$ are the Coriolis parameter; and $f r_{x}$ and $f r_{y}$ are frictional terms. This will have a solution if and only if 


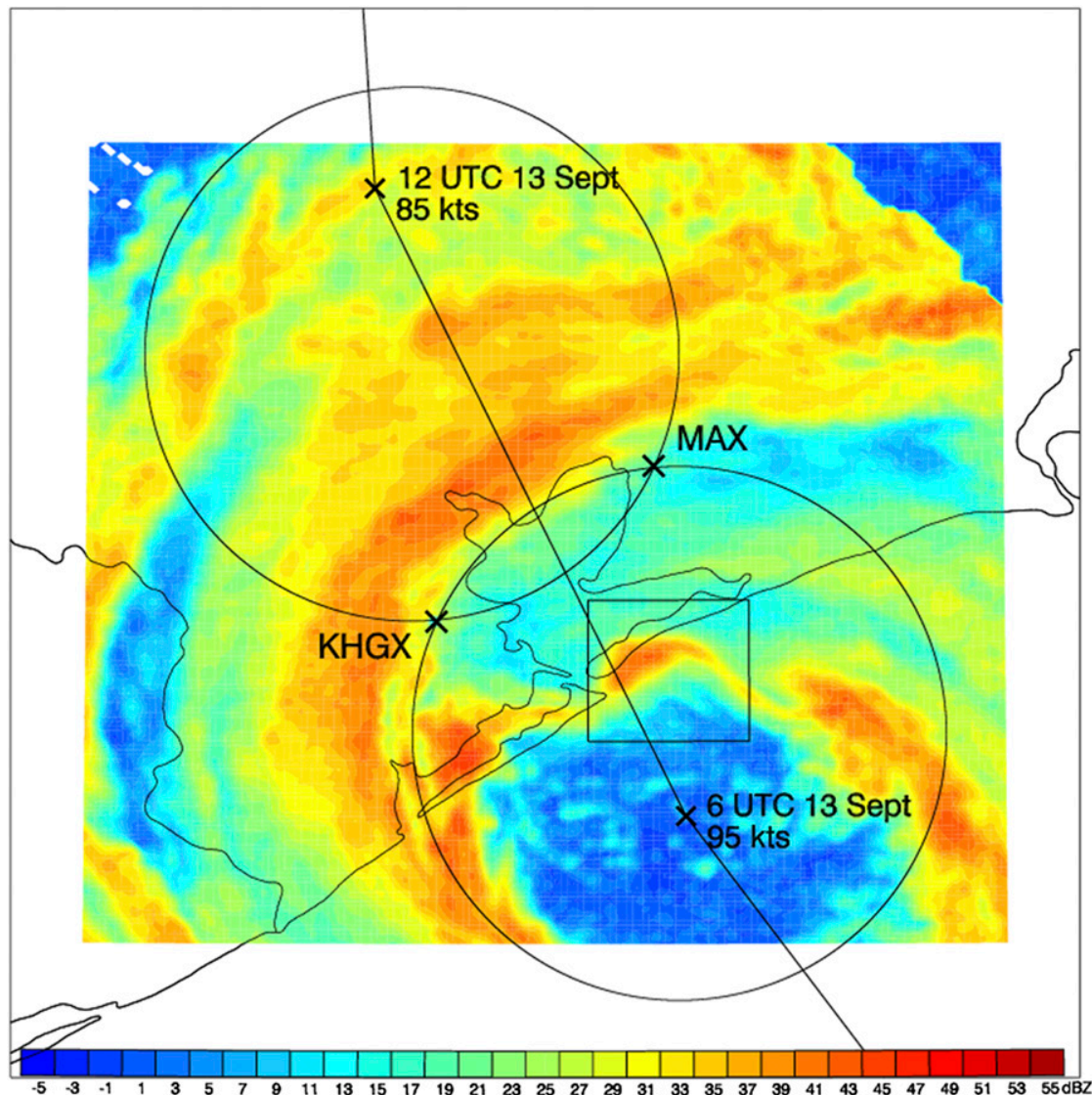

FIG. 3. Map of KHGX and MAX locations in southeast Texas with the best track path and intensity of Ike and outline of the dual-Doppler lobes shown. The baseline from MAX to KHGX is about $53 \mathrm{~km}$. The 0535 UTC $1.5-\mathrm{km}$ AGL KHGX reflectivity is shown across the larger $(160 \mathrm{~km} \times 160 \mathrm{~km})$ analysis domain. The black box shows placement of the smaller $30 \mathrm{~km} \times 30 \mathrm{~km}$ analysis domain for MV03 at this time.

$$
\frac{\partial F}{\partial y}=\frac{\partial G}{\partial x}
$$

In light of physical considerations (i.e., that the friction and derivative terms cannot be exactly determined), this is solvable only in a least squares sense. Doing so gives the Poisson PDE:

$$
\frac{\partial^{2} p^{\prime}}{\partial x^{2}}+\frac{\partial^{2} p^{\prime}}{\partial y^{2}}=\frac{\partial F}{\partial x}+\frac{\partial G}{\partial y}
$$

which is subject to Neumann boundary conditions. A local steady-state assumption is made to allow neglecting time derivatives, and friction is considered negligible. The values of $F$ and $G$ are calculated from the dual-Doppler-derived wind field and the Coriolis parameter. With $F$ and $G$ known, (3) is solved, obtaining estimated horizontal perturbation pressures. The retrieved values herein represent deviations from the mean pressure over the second, smaller domain, and are not to be considered perturbations from the minimum central pressure of Ike.
In total, 166 small domain dual-Doppler analyses were performed on 16 potential MV features that propagated through the dual-Doppler lobes during nearly $10 \mathrm{~h}$ of observation (about 0230-1215 UTC, Fig. 2).

\section{Eyewall evolution}

To provide context for the analysis presented in the remainder of this paper, we present a brief summary of Ike's eyewall evolution in the hours near landfall. Panels in

TABLE 1. KHGX and MAX radar volume scanning patterns.

\begin{tabular}{lc}
\hline \hline Pattern & Elevation angles $\left({ }^{\circ}\right)$ \\
\hline VCP 212 & $0.5,0.9,1.3,1.8,2.4,3.1,4.0,5.1$, \\
& $6.4,8.0,10.0,12.5,15.6,19.5$ \\
MAX1 & $0.5,0.8,1.0,1.5,2.0,2.5$ \\
MAX2 & $0.8,1.5,2.5,4.5,6.5,8.5,10.5,12.5,14.5,16.5$, \\
& $18.5,20.5,22.5,24.5,26.5,28.5,30.5$ \\
MAX3 & $0.8,1.5,2.0,2.5,3.5,4.5,5.5,6.5,8.0$, \\
& $9.5,11.0,13.0,15.0,17.0,19.0,21.0$ \\
\hline
\end{tabular}


TABLE 2. List of investigated features: dual-Doppler analysis time intervals, small domain size, and mean average peak values (1.5 km AGL) for various kinematic properties are given. The peak vertical vorticity value for each feature is indicated in parentheses. Feature names preceded by " $n$ " were not deemed MVs; feature names preceded by "i" are MVs that formed after Ike's center crossed the coastline; feature names shown in bold correspond to the detailed examples discussed in the text. The mean peak upward motion for all MVs (excluding nMV11 and nMV13) was $8.3 \mathrm{~m} \mathrm{~s}^{-1}$.

\begin{tabular}{|c|c|c|c|c|c|c|}
\hline Feature & $\begin{array}{l}\text { Analysis time } \\
\text { interval(s) } \\
\text { (UTC) }\end{array}$ & $\begin{array}{c}\text { Small } \\
\text { domain size }(\mathrm{s}) \\
(\mathrm{km} \times \mathrm{km})\end{array}$ & $\begin{array}{c}\text { Mean peak } \\
\text { (peak) } 1.5-\mathrm{km} \\
\text { vorticity }\left(\times 10^{-3} \mathrm{~s}^{-1}\right)\end{array}$ & $\begin{array}{c}\text { Mean peak } \\
\text { 1.5-km updraft } \\
\left(\mathrm{m} \mathrm{s}^{-1}\right)\end{array}$ & $\begin{array}{c}\text { Mean peak } \\
\text { 1.5-km downdraft } \\
\left(\mathrm{m} \mathrm{s}^{-1}\right)\end{array}$ & $\begin{array}{l}\text { Mean peak } \\
\text { 1.5-km horizontal } \\
\text { wind }\left(\mathrm{m} \mathrm{s}^{-1}\right)\end{array}$ \\
\hline MV01 & 0228 & $30 \times 30$ & $2.9(2.9)$ & 8.9 & 11.5 & 53.4 \\
\hline MV02 & $\begin{array}{l}0247-0304 \\
0433-0525 \\
0621-0650\end{array}$ & $30 \times 30$ & $4.9(8.5)$ & 8.9 & 10.5 & 50.5 \\
\hline MV03 & $\begin{array}{l}0304-0326 \\
0507-0554 \\
0654-0728 \\
0747-0808\end{array}$ & $\begin{array}{l}30 \times 30 \\
35 \times 35 \\
40 \times 40\end{array}$ & $5.7(8.2)$ & 9.1 & 9.1 & 54.0 \\
\hline MV04 & 0315-0336 & $30 \times 30$ & $4.5(4.8)$ & 7.6 & 10.3 & 55.1 \\
\hline MV05 & $\begin{array}{l}0336-0400 \\
0554-0612 \\
0713-0755 \\
0809-0900\end{array}$ & $\begin{array}{l}30 \times 30 \\
35 \times 35\end{array}$ & $5.5(9.6)$ & 6.7 & 7.2 & 54.9 \\
\hline MV06 & $0400-0424$ & $30 \times 30$ & $4.1(4.8)$ & 10.3 & 13.3 & 45.6 \\
\hline MV07 & $\begin{array}{l}0415-0440 \\
0559-0628 \\
0728-0819 \\
0848-0910\end{array}$ & $\begin{array}{l}30 \times 30 \\
40 \times 40\end{array}$ & $4.3(7.5)$ & 7.8 & 7.0 & 54.8 \\
\hline MV08 & 0433-0440 & $30 \times 30$ & $4.3(4.9)$ & 10.4 & 10.2 & 54.6 \\
\hline MV09 & $\begin{array}{l}0458-0535 \\
0630-0704 \\
0728-0738 \\
0801-0848 \\
0910-0933\end{array}$ & $\begin{array}{l}30 \times 30 \\
35 \times 35 \\
40 \times 40\end{array}$ & $5.0(8.0)$ & 8.1 & 7.6 & 54.8 \\
\hline MV10 & $\begin{array}{l}0525-0612 \\
0702-0738 \\
0809-0819\end{array}$ & $\begin{array}{l}30 \times 30 \\
40 \times 40\end{array}$ & $6.3(8.6)$ & 9.5 & 9.0 & 58.7 \\
\hline nMV11 & $0612-0630$ & $30 \times 30$ & $\mathrm{a}$ & 5.5 & 6.9 & 62.4 \\
\hline iMV12 & $\begin{array}{l}0704-0713 \\
0738-0747 \\
0838-0900 \\
0923-1025\end{array}$ & $30 \times 30$ & $4.3(6.5)$ & 6.6 & 5.8 & 51.1 \\
\hline nMV13 & 0809-0829 & $30 \times 30$ & $\mathrm{a}$ & 4.5 & 7.3 & 52.3 \\
\hline iMV14 & 0838-0949 & $30 \times 30$ & $4.7(6.0)$ & 5.9 & 5.1 & 52.9 \\
\hline iMV15 & $\begin{array}{l}0900-0910 \\
0949-1213\end{array}$ & $\begin{array}{l}30 \times 30 \\
35 \times 35\end{array}$ & $4.3(7.4)$ & 6.0 & 5.0 & 43.3 \\
\hline iMV16 & $\begin{array}{l}0900-0923 \\
1025-1112\end{array}$ & $30 \times 30$ & $4.3(5.4)$ & 10.6 & 6.3 & 38.7 \\
\hline
\end{tabular}

${ }^{\text {a }}$ The nMV11 and nMV13 did not contain distinct, localized cores of vertical vorticity and thus were not deemed MVs.

Fig. 1 show base elevation $\left(0.5^{\circ}\right) \mathrm{KHGX}$ radar reflectivity at hourly intervals, beginning about 0030 UTC. An animation of raw KHGX reflectivity and velocity for 0000 1300 UTC shows additional detail and is available in the online supplemental material.
The open eyewall seen at 0032 UTC (Fig. 1a) was undergoing an inward spiraling episode, not unlike the evolution discussed by Sitkowski and Barnes (2009). Over the next hour, a striking potential MV feature (annotated as MV06 because this entity became the sixth 
feature analyzed) led this progression, nearly completing the smaller eyewall by 0128 UTC (Fig. 1b). The potential MV06 feature advanced to the northernmost part of the eyewall, forming a vertex in a square-shaped eyewall at 0145 UTC, reduced to a triangular arrangement by 0229 UTC (Fig. 1c). Dual-Doppler sampling of Ike's MVs first occurred at this time for the northern vertex (MV01).

For about the next $10 \mathrm{~h}$ as Ike advanced, potential MV features within its eyewall traversed the dual-Doppler domain. By 0331 UTC, several potential MVs (labeled in Fig. 1d) had formed as the shape of the eyewall continued to change. By 0430 UTC, the eyewall assumed an oblate hexagonal shape, suggesting the presence of six possible MVs, two fully within in the analysis domain (Fig. 1e). Over the next hour, the best radar-relative geometries for wind syntheses in the southeastern dual-Doppler lobe occurred, as shown at 0530 UTC in Fig. 1f with MV03 situated near the heart of the lobe, MV09 downstream, and MV10 upstream. The MV entities advanced cyclonically about the eye as Ike approached the coast. By 0631 UTC (Fig. 1g), the eyewall took on a more symmetric hexagonal shape, approximately aligned along the southeastern lobe.

Ike's center crossed the Bolivar Peninsula at 0700 UTC, and within about $0.5 \mathrm{~h}$ the hexagonal eyewall shape again elongated, with MV09 and MV03 just entering the northwest analysis lobe and MV10 and MV05 in the southeastern lobe (Fig. 1h). At 0828 UTC (Fig. 1i), the eyewall straddled the dual-Doppler domain, and from this point on the northwest lobe became the main analysis region. In the hours after landfall, eyewall polygons became less discernable, but distinct curl features were still evident in reflectivity, as for MV09, iMV12, iMV14, iMV15, and iMV16 at 0929 UTC (Fig. 1j). After this time, the eyewall structure became increasingly obscured, and MVs were less obvious in reflectivity (but still discernable as local $\zeta$ maxima in the large and small grid analyses). By 1029 UTC (Fig. 1k), only a few remained in the analysis region. The northernmost part of Ike's remnant eyewall began exiting the dual-Doppler region after 1100 UTC (Fig. 11). In total, the analysis interval was about 10 h: 0228 (first analysis of MV01) to 1213 UTC (final analysis of iMV15, see Table 2).

\section{Dual-Doppler results}

Summarized results for each investigated feature are listed in Table 2. Only entities demonstrating a clear local low-level (here, $1.5 \mathrm{~km}$ ) $\zeta$ maximum are deemed MVs; all but two (nMV11 and nMV13) of the examined features meet this criterion. Many of the MVs persist for $5+\mathrm{h}$ and complete two full revolutions about the eye as they progress counterclockwise. Representative results from three specific analysis times are shown in sections $4 a-c$; we note that the trends presented for these features are consistent with the other MVs studied. Horizontal, plan view analysis plots at $1.5 \mathrm{~km}$ are shown to ensure the most complete sampling by both radars.

The most significant finding of this study is that the arrangement of $\zeta$ and $w$ maxima within each MV progresses in a common fashion as the MVs are located in various regions of the eyewall. After Ike's center crossed the Bolivar Peninsula, MVs continued to persist and even initiate. The MVs forming after landfall (0700 UTC) are denoted inland MVs, or iMVs. Interaction with the coastline and changes to the hurricane boundary layer as the storm moved inland and began weakening would have modified the processes generating and maintaining the MVs (e.g., Wu et al. 2009). Early on, iMVs exhibit characteristics akin to MVs that formed while at least half of the eyewall was still over the Gulf of Mexico, but as Ike moves inland obvious departures from these persistent kinematic arrangements occur.

Results for three representative MV analysis times are presented below. These were selected to show 1) the consistent, characteristic arrangements of kinematic fields identified in prelandfall MVs; 2) the breakdown of towerlike structures of enhanced $\zeta$ after landfall; and 3) an example of departures from the characteristic kinematic arrangements after the inner core experienced prolonged interaction with the rougher, land surface. A visual summary of the consistent low-level kinematic structures, prior to prolonged land interaction, and a depiction of the demise of the vertical structure of the $\zeta$ field as Ike progressed inland are given as well.

\section{a. MV03 at 0535 UTC}

The four time intervals listed in Table 2 for MV03 analyses correspond to periods when the feature traversed either the southeastern or northwestern dual-Doppler lobe. The smaller domain indicated in Fig. 3 is set over MV03 in the northern eyewall during its second pass through the lobes. This time, 0535 UTC, marked the best geometric location of MV03 relative to the radars for dual-Doppler retrieval. Results for this time are shown in Fig. 4. The $1.5-\mathrm{km} \zeta$ field shows a distinct $6.2 \times 10^{-3} \mathrm{~s}^{-1}$ maximum toward the forward, or downstream, portion of the arced reflectivity signature [black " $X$ ", in Fig. 4a at approximately $(20,-33)]$. Upward $w$ values peak at $6.8 \mathrm{~m} \mathrm{~s}^{-1}$ nearby [gray " $\times$ " in Fig. 4a at about $(19,-31)]$, with the most intense portion of the updraft located radially outward and northward from the $\zeta$ peak.

Additional kinematic fields at this time are consistent with the presence of a $\zeta$ maximum and paint a harmonious picture of the MV's impact on the primary 
(a)
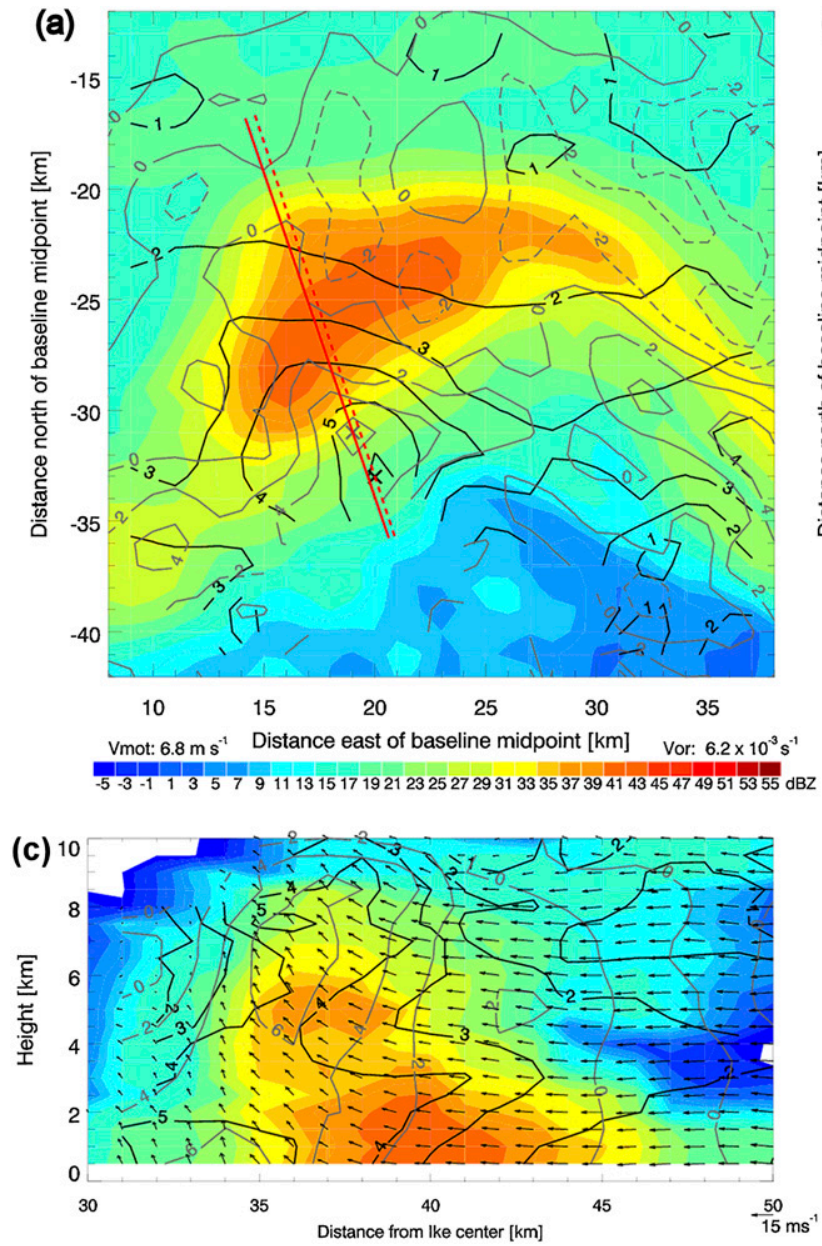

cyclonic flow and secondary circulation of the hurricane. Figure $4 \mathrm{~b}$ shows enhanced convergence in toward the MV center, and a strong divergent signature out ahead (downstream) of the $\zeta$ peak. This arrangement occurs in nearly all of the MVs: locally enhanced convergence toward the MV on the upstream side of the local $\zeta$ maximum, and divergence toward the front, downstream of the MV $\zeta$ center. A shift in the hurricane's radial wind component occurs in line with the MV location, with radial inflow up to $20 \mathrm{~m} \mathrm{~s}^{-1}$ in toward the storm center found at the downstream side of the $\mathrm{MV}$, and radial outflow peaking at $15 \mathrm{~m} \mathrm{~s}^{-1}$ away from the storm center on the MV's upstream side. This pattern is evident in nearly all of the MVs prior to significant land interaction. Emanuel (1991) provides a typical hurricane radial velocity scale as on the order of $10 \mathrm{~m} \mathrm{~s}^{-1}$; observations reported by Barnes and Powell (1995) and Wroe and Barnes (2003) show radial flow magnitudes up to about $20 \mathrm{~m} \mathrm{~s}^{-1}$. In comparison to previous work, radial wind component magnitudes in our results are somewhat, but not drastically, large.

Retrieved perturbation pressure values are generally aligned with the eyewall, approximately perpendicular to

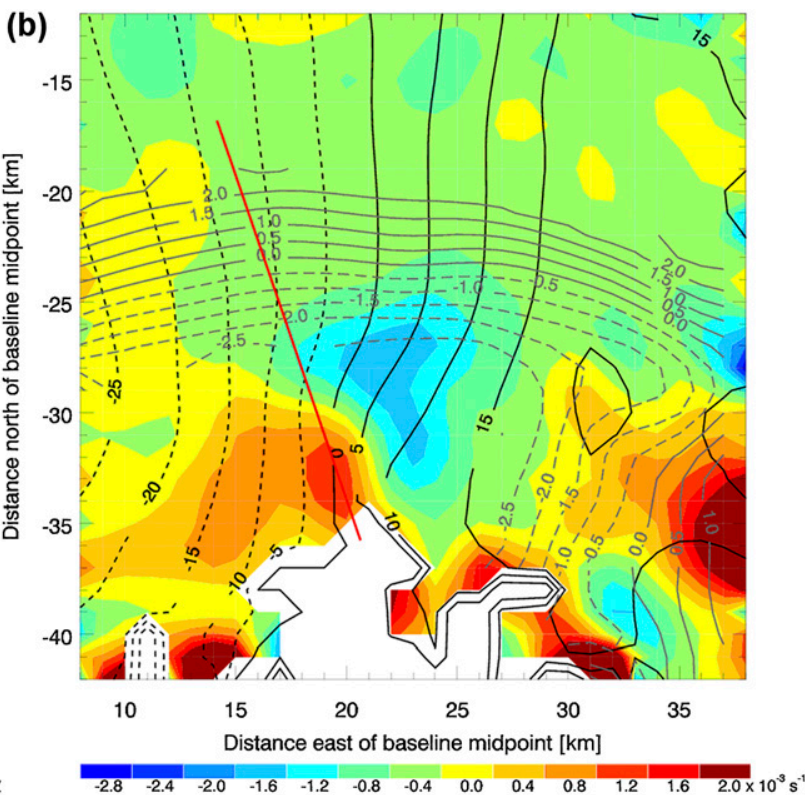

FIG. 4. The 0535 UTC small domain (see Fig. 3) analysis results for MV03 in the northern eyewall. (a) The $1.5-\mathrm{km}$ reflectivity (shaded), $\zeta$ (black, $\times 10^{-3} \mathrm{~s}^{-1}$ ), and $w$ (gray, $\mathrm{m} \mathrm{s}^{-1}$ ); locations of maximum $\zeta(w)$ values are indicated with a black (gray) " $\times$." (b) The 1.5-km divergence (shaded), perturbation pressure (gray, $\mathrm{hPa}$ ), and radial wind component (black, $\mathrm{m} \mathrm{s}^{-1}$ ). (c) Ike-relative radius-height vertical cross section along the red line indicated in (a),(b); reflectivity (shaded), $\zeta$ (black, $\left.\times 10^{-3} \mathrm{~s}^{-1}\right), w\left(\right.$ gray, $\mathrm{m} \mathrm{s}^{-1}$ ), and wind vectors (a $15 \mathrm{~m} \mathrm{~s}^{-1}$ scale vector is shown below the range axis). Panels (a) and (c) use the same reflectivity color scale.

the radial wind, as one would expect for a barotropic system. We note again that the perturbations here are relative to the mean pressure over the entire small domain (area shown in Figs. 4a and 4b), and should not be considered as perturbations from the central pressure of the hurricane. Even with this caveat, results suggest a localized pressure perturbation minimum in the vicinity of the MV0 $\zeta$ peak, supported by the sharp departure from the broad curve of the eyewall in the eastern part of domain and the nearly closed $-2.5-\mathrm{hPa}$ contour outlining the center of the feature.

The Ike-relative radius-height vertical cross section in Fig. $4 \mathrm{c}$ intersects the low-level $w$ maximum; it is taken along the red line indicated in Figs. $4 \mathrm{a}$ and $4 \mathrm{~b}$ and shows the flow in that plane with wind vectors along with $\zeta$ and $w$ contours. We note that a similar view through the lowlevel $\zeta$ maximum produces a nearly identical image (not shown) as the two points are spatially very close (black and gray " $\times$ " locations in Fig. 4a). The largest $\zeta$ values $(6.0 \times$ $10^{-3} \mathrm{~s}^{-1}$ ) are found at the lowest level in the analysis domain $(0.5 \mathrm{~km})$. While the largest $\zeta$ is confined to below $2 \mathrm{~km}$, values of $4 \times 10^{-3} \mathrm{~s}^{-1}$ extend above the 8 - $\mathrm{km}$ level, indicating a towerlike structure. This tower contains the 
maximum $w$ values and a secondary $\zeta$ peak, seen as the closed $5 \times 10^{-3} \mathrm{~s}^{-1}$ contour just downstream of the $7.6 \mathrm{~m} \mathrm{~s}^{-1} w$ maximum. The enhanced $\zeta$ aloft can be ascribed to stretching of the column by the updraft, tilting due to the inward component of the wind, as well as vertical advection of the large low-level $\zeta$. Similar towerlike structures of enhanced $\zeta$ aloft exist for the mature MVs, especially prior to prolonged land interaction.

\section{b. MV09 at 0838 UTC}

A second example shows the typical progression of updraft location for MVs in a different region of Ike's eyewall and changes in the characteristic arrangements as the hurricane moved inland. For about the first hour of landfall (0700-0800 UTC), MV kinematic structures maintain the patterns seen in MVs that developed while the TC center was still offshore. Preferential updraft locations continue to progress around the $\zeta$ maximum. As parcels within the inner core boundary layer spent time over land, the characteristic MV kinematic patterns began to transition. While distinct $\zeta$ centers become more elongated and arc shaped, they persist as obvious local maxima. The perturbation pressure signature is the first to be lost as the hurricane moves inland, and the pattern of convergence and divergence becomes attenuated after more than $1 \mathrm{~h}$ of land interaction. In the vertical, the towerlike structure of enhanced $\zeta$ decreases to typical heights of about $4 \mathrm{~km}$ (with peak magnitudes falling from about $7 \times 10^{-3}$ to about $4 \times 10^{-3} \mathrm{~s}^{-1}$ ).

Figure 5a shows the location of MV09 in the southeastern eyewall (along the Bolivar Peninsula coast) at 0838 UTC and placement of the $30 \mathrm{~km} \times 30 \mathrm{~km}$ analysis domain. The remaining panels in Fig. 5 show results for this time, about $1.5 \mathrm{~h}$ after landfall. As Ike moves inland, the bull's-eye configuration of the low-level $\zeta$ field in the MV transitions to a more elongated structure, as shown in the Fig. 5b example. Vertical motion maxima maintain the same relative positions to the $\zeta$ peak as occurred prior to landfall. In Fig. 5b, the black and gray " $X$ " symbols mark the locations of the $\zeta$ and $w$ maxima, at about $(21,-27)$ and $(18,-39)$, respectively. Even though the largest contours of $\zeta$ and $w$ appear slightly less distinct (e.g., less of a bull's-eye than in Fig. 4), the relative locations of the largest $\zeta$ and $w$ for MV09 at this time match that of other MVs in the southeastern eyewall: the $w$ maximum is positioned radially outward and to the south of the $\zeta$ peak. More obvious departures from the characteristic MV structure can be seen in Fig. 5c. While the sign change in the radial wind component is absent, there is still enhanced radial inflow at the downstream side of the MV. The signature convergence near the middle of the MV is present; however, there is no prominent divergence region at the MV's downwind side as found for
MVs prior to landfall. Suggestion of a local minimum in the perturbation pressure is lacking.

In the vertical, the $\zeta$ values are less intense and do not extend as high aloft as they did before landfall. This is evident in the MV09 example at 0838 UTC, with Ikerelative radius-height cross sections through the lowlevel $\zeta$ (Fig. 5d) and $w$ (Fig. 5e) peaks. Both panels depict a vertical structure of locally enhanced $\zeta$ (with largest values at heights below about $2 \mathrm{~km}$ ), but the relatively high values are confined (a closed $4 \times 10^{-3} \mathrm{~s}^{-1}$ contour extends up to only $4 \mathrm{~km}$ ) in contrast to that diagnosed in MV03 when the maximum $\zeta$ and $w$ contours were also more distinct, highlighted in section $4 \mathrm{a}$. The vertical towerlike structure of the maximum $\zeta$ seen at earlier times begins to wane in both intensity and vertical extent as Ike continues inland. Breakdown of the towerlike structures of enhanced $\zeta$ after landfall was analyzed in all MVs that developed before landfall and dissipated during or after landfall as shown in further detail below.

Even more than $1 \mathrm{~h}$ after landfall, the relative horizontal positions of the $w$ and $\zeta$ maxima remain consistent with those diagnosed at earlier times. For all investigated MVs that formed prior to landfall, the initial deterioration of the prelandfall kinematic arrangements generally follows the pattern as shown for MV09: the perturbation pressure and radial wind signatures depart first, then the $\zeta$ maximum becomes confined to lower levels. As shown in the next example, after multiple hours of land interaction, the characteristic convergence/ divergence configuration also becomes convoluted.

\section{c. $i M V 12$ at 1001 UTC}

An example of the drastic departures from the structure of the mature MVs before and just after landfall can be seen in the erratic organization of iMV12 about $3 \mathrm{~h}$ after landfall. Figure 6 a shows the location of the entity within the western eyewall and the placement of the $30 \mathrm{~km} \times$ $30 \mathrm{~km}$ analysis domain at 1001 UTC. Results for the dualDoppler and perturbation pressure derivations are shown in Figs. $6 \mathrm{~b}$ and $6 \mathrm{c}$. The distribution of $\zeta$ within the MV reveals multiple local maxima: while the low-level values are still somewhat enhanced [up to $6.3 \times 10^{-3} \mathrm{~s}^{-1}$, located at about $(-44,22)]$, they are not organized in a single, coherent core but exist as multiple, cellular components. Arrangements of the $w$ field also do not strictly emulate the patterns exhibited by the earlier MVs. The updraft to the southwest of the three $\zeta$ maxima is situated in the preferred updraft area seen for MV in the western eyewall prior to extended land interaction (radially outward and southwest of the $\zeta$ peak); however, a second, similar magnitude $w$ peak (both $w$ maxima are on the order of $5 \mathrm{~m} \mathrm{~s}^{-1}$ ) is located to the north of the high $\zeta$ area [near $(-49,35)$ in Fig. 6b]. Other analyzed fields are more chaotic. There is no clear 

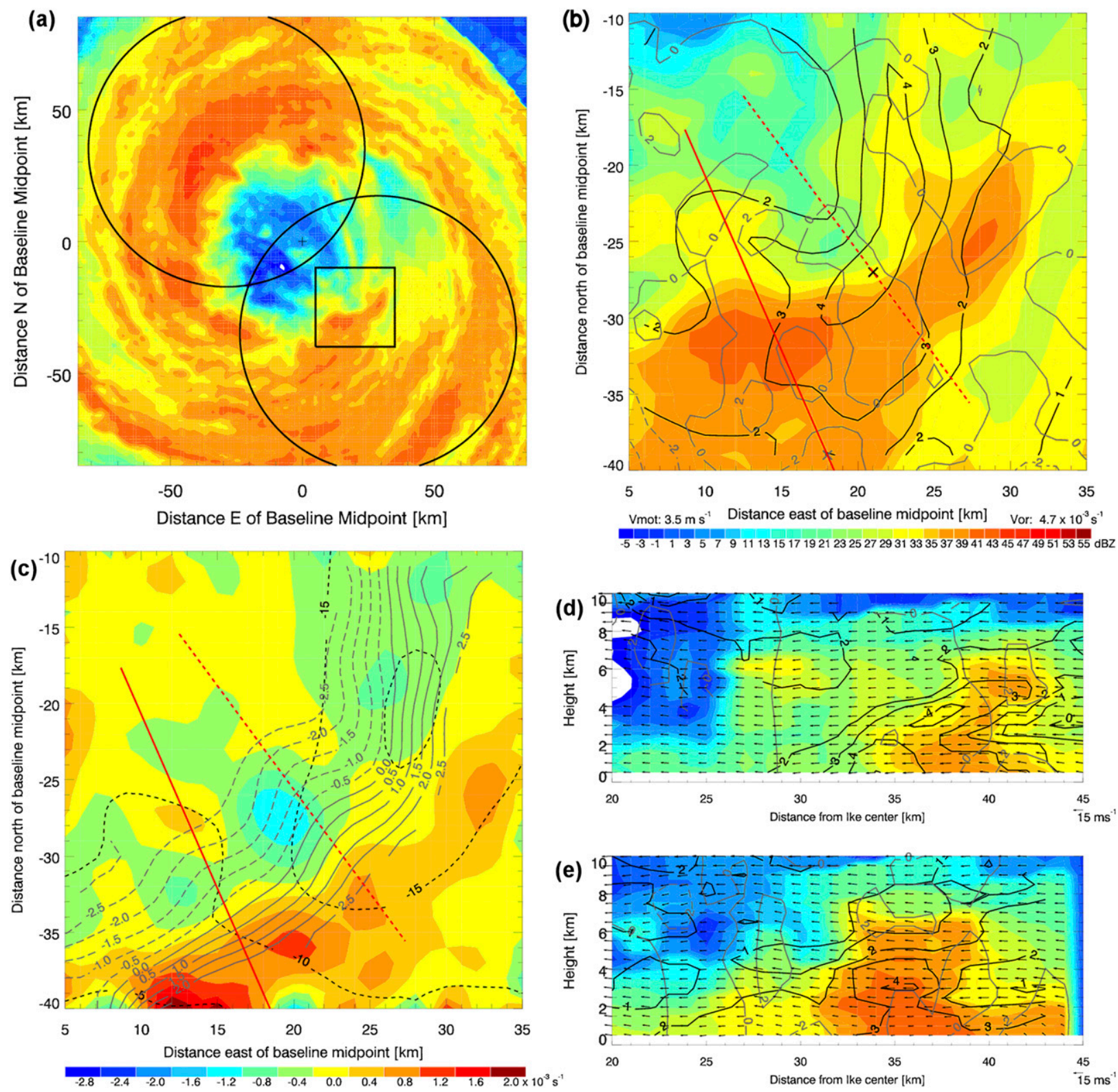

FIG. 5. (a) The 0838 UTC small domain location and analysis results for MV09 in the southeastern eyewall. (b) As in Fig. 4a, and (c) as in Fig. 4b. The red dashed and solid lines locate the Ike-relative radius-height vertical cross section intersecting the low-level (d) $\zeta$ and (e) $w$ maxima. The fields in (d) and (e) are as in Fig. 4c. Panels (a),(b),(d), and (e) use the same reflectivity color scale.

convergence/divergence pattern comparable to that evident at previous MV analysis times, and the perturbation pressure and radial wind component contours have become erratic. Not only has the direction of the radial flow shift been reversed (e.g., radial outflow downwind and radial inflow upwind, suggesting a possible deformation pattern), but the perturbation pressure contours zigzag across iMV12 in a configuration not seen in any of the MV structures prior to multiple hours of land interaction. Ike-relative radius-height cross sections through the low-level $\zeta$ and $w$ peaks (Figs. 6d,e) depict the continued demise of the towerlike structures of enhanced $\zeta$. Broadly similar, chaotic kinematic patterns in the low-level horizontal plane, as well as degraded $\zeta$ magnitudes in the vertical direction, were also diagnosed for iMVs 14, 15, and 16 as Ike progressed inland.

\section{d. Summary of general MV kinematic structure}

Results from nearly $10 \mathrm{~h}$ of dual-Doppler analysis of Ike's MVs reveal persistent kinematic structures prior to 


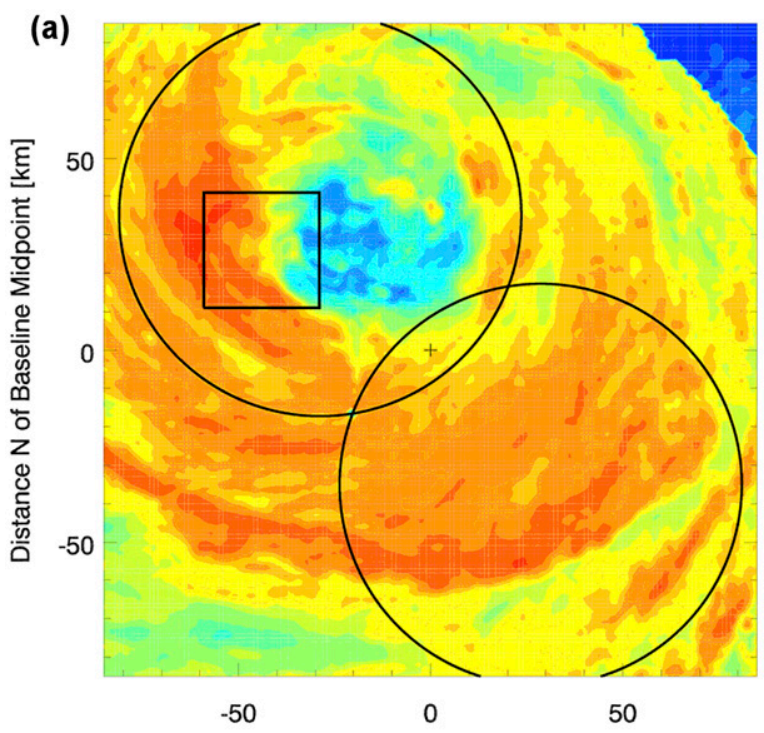

Distance $\mathrm{E}$ of Baseline Midpoint $[\mathrm{km}]$

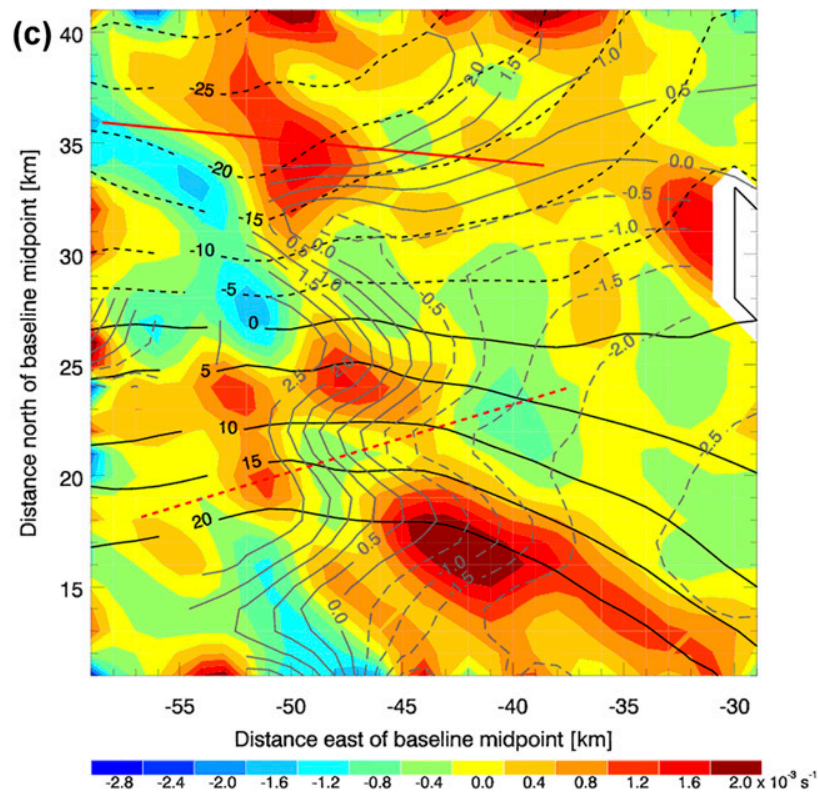

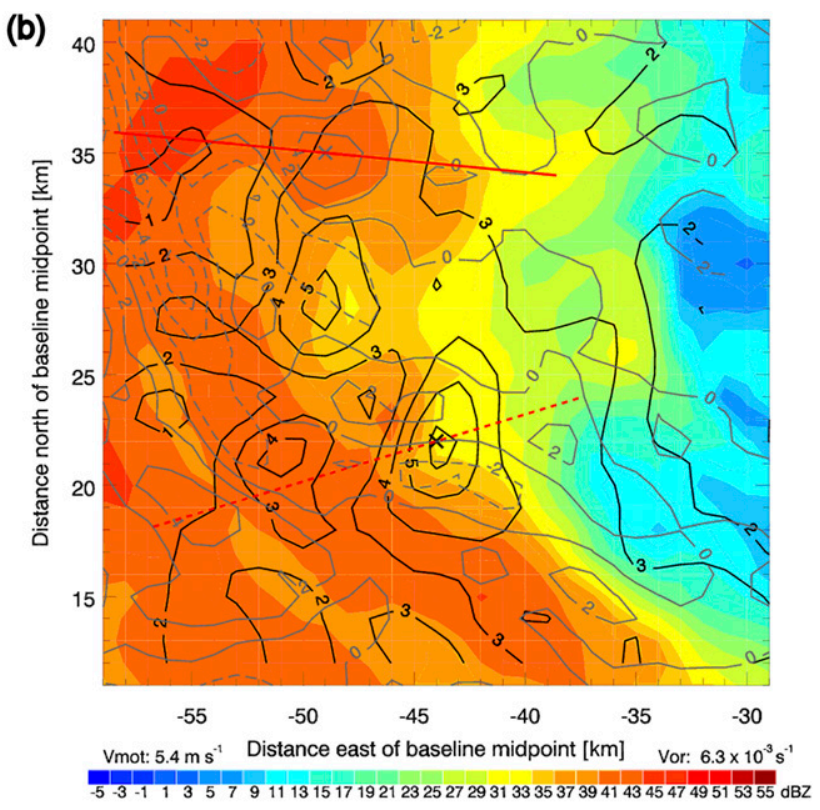
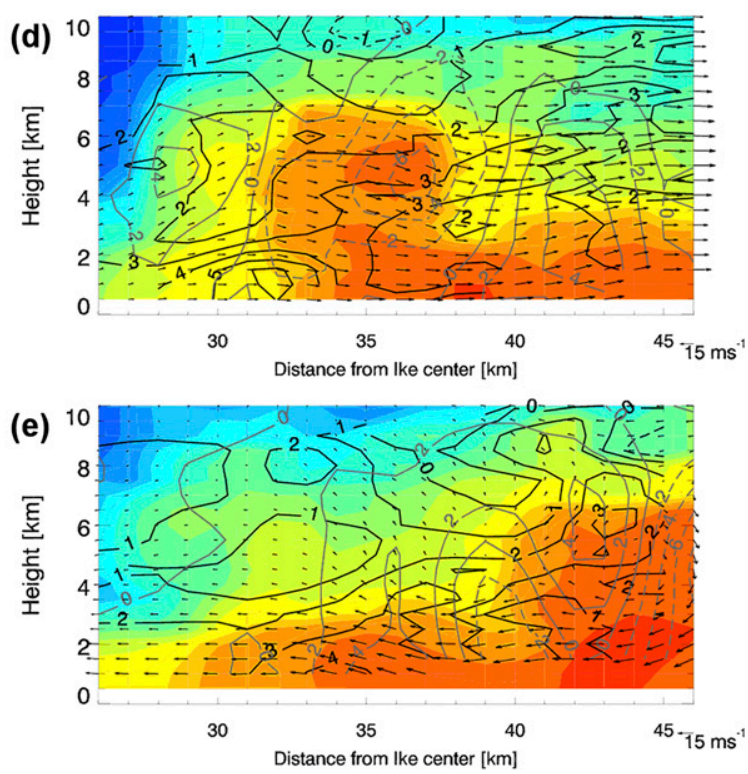

FIG. 6. (a) The 1001 UTC small domain location and analysis results for iMV12 in the western eyewall. (b) As in Fig. 4a, and (c) as in Fig. 4b. The red dashed and solid lines locate the Ike-relative radius-height vertical cross section intersecting the low-level (d) $\zeta$ and (e) $w$ maxima. The fields in (d) and (e) are as in Fig. 4c. Panels (a),(b),(d), and (e) use the same reflectivity color scale.

significant land interaction. The schematic presented in Fig. 7 summarizes the characteristic low-level horizontal kinematic arrangements observed in Ike's eyewall MVs prior to significant land interaction. Several factors have been shown to affect TC inner core structures (e.g., Braun et al. 2006; Braun and Wu 2007; Wu et al. 2009; Nguyen and Molinari 2015). Thus, we note that this schematic conveys results for the Ike case; TC eyewall MVs in other instances may display variations on the structures revealed by our study. The MV structure is marked in reflectivity by an arc-shaped echo of $30+\mathrm{dBZ}$. The MV $\zeta$ maximum is most often located toward the front (downstream side) of the reflectivity arc; a few of Ike's MVs contain $\zeta$ centers more in the middle of the arc. Existence of these localized $\zeta$ maxima were used in this study to determine if features of interest were indeed MVs (see Table 2). As the intervals listed in Table 2 indicate, a temporally continuous set of analyses for the entire lifetime of a given MV was not possible (due to dual-Doppler geometry and the movement of the $\mathrm{MVs}$ ), thus, it is difficult to diagnose the time scale of 


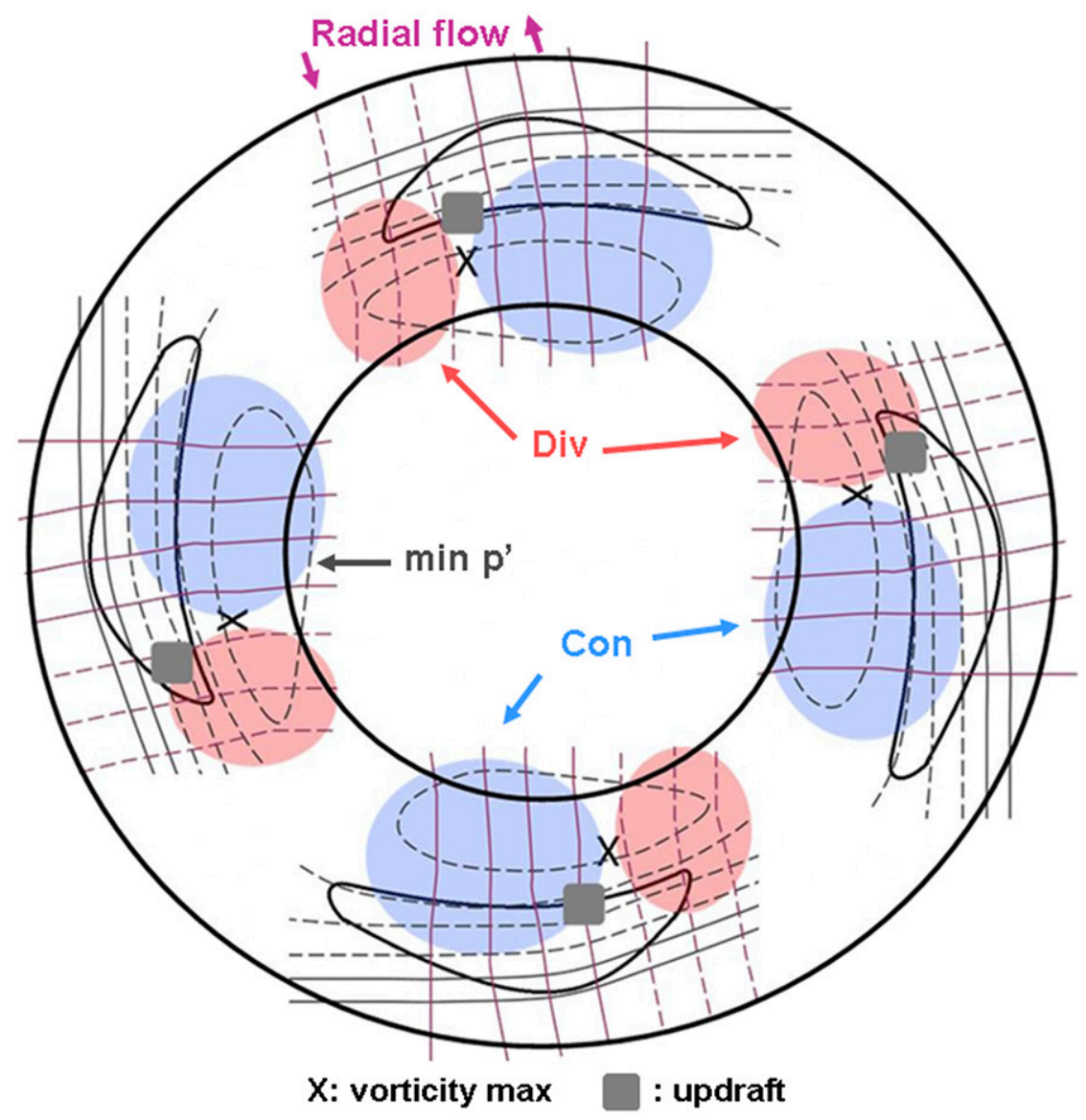

FIG. 7. Schematic summarizing the low-level horizontal structure of Ike's eyewall MVs. Representation of MVs in cardinal regions of Ike's eyewall (concentric black circles). Solid black outlines represent the $30-\mathrm{dB} Z$ curved reflectivity signature, $\times \mathrm{s}$ show the location of local low-level vertical vorticity maxima, and gray squares indicate preferential updraft areas. Annotations highlight areas of divergence (convergence) in red (blue), the radial wind component shift (violet lines), and suggested local perturbation pressure minima (gray lines).

the progression of the updrafts' preferential location with certainty. However, our results show that for MVs sampled in nearly all cardinal and ordinal regions of the eyewall, updrafts are consistently situated on the radially outward side of the MV $\zeta$ maximum, appearing to rotate counterclockwise about the $\zeta$ peak as the MV features themselves revolve around the eye. Regions of convergence and divergence flank either side of the MV $\zeta$ center, and retrieved perturbation pressure values suggest a local pressure minimum. A shift in the direction of the hurricane's radial wind component occurs about the $\zeta$ peak in mature MVs. After a majority of Ike's inner core region crosses the Texas coastline (recall that landfall occurred at about 0700 UTC), these characteristic arrangements begin to deteriorate, as shown in the examples detailed above.

The most striking vertical structures in Ike's MVs are the relatively deep, towerlike formations of enhanced $\zeta$.
Mature MVs (e.g., MV03 at 0535 UTC, Fig. 4) contain the largest $\zeta$ in the lowest levels ( $2 \mathrm{~km}$ and below), a mostly vertical contour of at least $4 \times 10^{-3} \mathrm{~s}^{-1}$ extending above $8 \mathrm{~km}$ AGL, and a secondary peak aloft, generally about $5 \times 10^{-3} \mathrm{~s}^{-1}$ located near $8 \mathrm{~km}$. The largest $\zeta$ values typically are found in the low levels, even after the vertical extent of the tower structures begins to degrade. Once the secondary $\zeta$ peak aloft weakens, the vertical extent of the $4 \times 10^{-3} \mathrm{~s}^{-1}$ contour decreases until eventually enhanced values aloft are limited to a smaller magnitude secondary peak at about $4-6 \mathrm{~km}$, with little vertical extent above that height. Figure 8 shows the time versus height progression of the $\zeta$ field for the MVs with the most dual-Doppler sampling times. The line segments show $1.5-\mathrm{km}$ peak $\zeta$ values for each available analysis interval, plotted on the ordinate with units as $\times 10^{-3} \mathrm{~s}^{-1}$. Numerals in these plots indicate the magnitude of the $\zeta$ peak aloft, which 

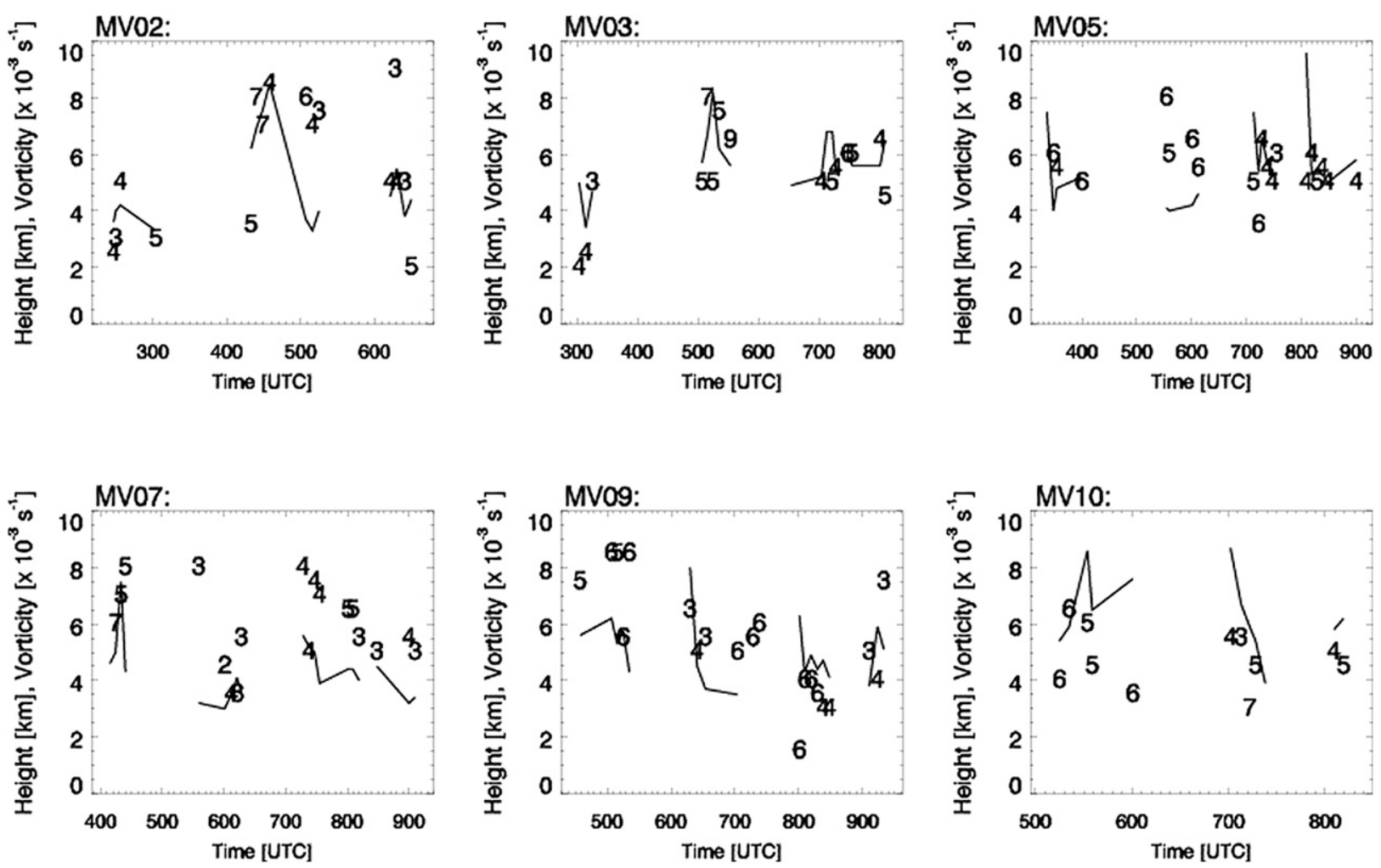

FIG. 8. Distribution of vertical vorticity with time for Ike's eyewall MVs with the longest dual-Doppler sampling time intervals. Peak value of vorticity aloft (magnitude rounded to nearest $\times 10^{-3} \mathrm{~s}^{-1}$ and plotted as numerals using the ordinate in $\mathrm{km}$ AGL units) and $1.5-\mathrm{km}$ vertical vorticity values (lines, plotted using the ordinate in $\times 10^{-3} \mathrm{~s}^{-1}$ units) for the MV noted at the top left of each panel.

occurs at the height where the numeral is placed on the ordinate, treating the units as $\mathrm{km} \mathrm{AGL.} \mathrm{After}$ 0600-0700 UTC (the 1-h period prior to landfall), the magnitude of $\zeta$ aloft in the MVs decreases and is typically located at a lower height. The most robust $\zeta$ tower signatures are found prior to significant interaction of Ike with the coastline and rougher land surface. Low-level MV $\zeta$ maxima magnitudes display a wider range and do not have as clear a decrease as noted for the aloft peaks.

As Ike moved inland and weakened, the eye gradually became obscure (Fig. 1). Interactions between the hurricane and MVs with land, presumably due to changes in the boundary layer resulting from increased surface roughness and less available warm water, are likely to have modified the dynamics that govern MV formation and maintenance. Deterioration of the towerlike $\zeta$ structures and the chaotic kinematic field patterns found at the later analysis times (e.g., iMV12 at 1001 UTC) indicate the demise of the entities and warrant further study on the physical mechanisms during MV episodes and landfall that alter the structure and sustenance of TC inner core MVs.

\section{Discussion}

Prior to multiple hours of land interaction, the observed arrangements of the MV kinematic fields are consistent. The preferential location of the low-level $w$ maximum rotates cyclonically with time about the low-level $\zeta$ peak as the MVs themselves progress cyclonically in the eyewall (Fig. 7). Magnitudes of these MV $w$ peaks are largest when the entity is located on the upshear side of the hurricanethe north-northeast portion of the eyewall (see the shear vector direction shown in Fig. 1d). Table 3 demonstrates this effect by listing representative updraft values in MVs located in the cardinal and ordinal regions of the eyewall, with the exception of the southwest region as it was not as well sampled in the dual-Doppler lobes. Based on high-resolution numerical simulation of Hurricane Bonnie (1998), Braun et al. (2006) developed a conceptual framework showing enhanced convergence and vertical motion resulting from interactions between shear-induced lowlevel flow and eyewall MVs on the downshear side of the hurricane (their Fig. 18), and similar results were obtained for the case of Hurricane Erin (2001) when that storm experienced weak to moderate vertical shear (Braun and $\mathrm{Wu}$ 2007). While our results are inconsistent with the 
TABLE 3. Representative values of $1.5-\mathrm{km}$ vertical vorticity $\left(\times 10^{-3} \mathrm{~s}^{-1}\right)$ and upward vertical velocity $\left(\mathrm{m} \mathrm{s}^{-1}\right)$ associated with MVs in the cardinal and ordinal regions of the eyewall (the SW region is excluded as it was not as well sampled in the dual-Doppler lobes). Strongest updrafts occur for MVs located in the upshear (north-northeast) side of the eyewall.

\begin{tabular}{cccccc}
\hline \hline $\begin{array}{c}\text { Time } \\
\text { (UTC) }\end{array}$ & MV & $\begin{array}{c}\text { Eyewall } \\
\text { region }\end{array}$ & $\begin{array}{c}\text { Updraft location } \\
\text { relative to } \\
\text { vorticity max }\end{array}$ & $\begin{array}{c}\text { Vorticity } \\
\left(\times 10^{-3} \mathrm{~s}^{-1}\right)\end{array}$ & $\begin{array}{c}\text { Updraft } \\
\left(\mathrm{m} \mathrm{s}^{-1}\right)\end{array}$ \\
\hline 0630 & 02 & E & N & 5.5 & 6.1 \\
0424 & 07 & NE & E & 5.0 & 10.8 \\
0535 & 03 & N & NW & 6.2 & 6.8 \\
0829 & 05 & NW & W & 5.5 & 4.2 \\
0910 & 14 & W & SW & 6.0 & 5.8 \\
0728 & 05 & S & SW & 6.6 & 5.8 \\
0838 & 09 & SE & S & 4.7 & 3.5 \\
\hline
\end{tabular}

conceptual model, we note that, in contrast to the Braun et al. (2006) and Braun and Wu (2007) simulation studies where the storm is entirely over open water, the present Ike case includes interactions with the coast and rougher land surface, which would induce additional asymmetries in the low-level flow that differ from a more purely sheardriven asymmetry.

The largest updraft magnitudes for all the MVs documented here surpass previous statistical studies' results for typical hurricane vertical motion values. Black et al. (1996) used airborne Doppler velocity data from seven Atlantic hurricanes to show that over $70 \%$ of vertical motions fell in the $\pm 2 \mathrm{~m} \mathrm{~s}^{-1}$ range, and only about $5 \%$ of eyewall vertical velocities exceeded $5 \mathrm{~m} \mathrm{~s}^{-1}$. Extensive observations by Jorgensen et al. (1985) showed a similar distribution. Based on this documentation of vertical motion magnitudes, our results indicate the dual-Doppler-retrieved vertical motions in Ike's MVs are well within the top $5 \%-10 \%$ of hurricane vertical velocities.

Perturbation pressure retrievals across the smaller analysis domains suggest the presence of localized pressure minima in Ike's MVs, mainly prior to prolonged land interaction. These results are corroborated by evidence from previous studies. Numerical simulations presented by Kossin and Schubert (2001) showed that MVs can develop local pressure minima, and observations of MVs in Hugo (Marks et al. 2008), Andrew (Willoughby and Black 1996), and Isabel (Aberson et al. 2006) documented pressure values in MVs that were lower than the central pressure of the primary vortex. While the methodology employed here to arrive at the perturbation pressure values requires the results only be considered as departures from the mean across the small analysis domains, not from Ike's central pressure, the pattern shown is consistent with a localized minimum.

Even beyond an hour after landfall, the preferred horizontal locations of the $w$ maxima relative to the $\zeta$ peak are maintained, though the shape of the $\zeta$ peak transitions to an elongated form. A convergence-divergence pattern akin to that seen in prelandfall MVs persists, but the shift in radial wind component across the MV and the perturbation pressure patterns are first to be lost, and the vertical extent of the enhanced $\zeta$ tower structure is limited. After multiple hours postlandfall, all of the consistent low-level kinematic field patterns in the horizontal plane decay. From the radar-based analysis, it is unclear whether the deterioration of the distinct $\zeta$ tower structures (Fig. 8) occurs in response to land interaction, MV processes governing its demise, or both.

The largest $\zeta$ is consistently confined to the lowest $2 \mathrm{~km}$, consistent with the results of Hendricks and Schubert (2010), Hendricks et al. (2012), and Menelaou et al. (2013). Braun et al. (2006) showed that MV updrafts can act to strengthen local $\zeta$ via stretching, and thereby constantly replenish a high $\zeta$ annulus, aiding the maintenance of existing MVs and the generation of new ones. Rozoff et al. (2009) noted the importance of this annulus replenishment as a driver for vorticity mixing episodes. Our results depicting towerlike structures of enhanced $\zeta$ in Ike's MVs suggest this type of rebuilding. These structures also suggest a resemblance to the more unstable regime 1 discussed by Kossin and Eastin (2001). As Ike moves inland and as the MVs weaken, largermagnitude $\zeta \geq 4 \times 10^{-3} \mathrm{~s}^{-1}$ contours do not extend much above $4 \mathrm{~km}$. Thus, if the MVs in Ike indeed developed and were maintained in response to the breakdown, mixing, and replenishment of a barotropically unstable high $\zeta$ annulus, as current $\mathrm{MV}$ theories would suggest, then the degradation of the towerlike $\zeta$ structures is consistent with the decay in the other characteristic MV structures observed here (elongation of the local low-level $\zeta$ peak and disappearance of the radial flow shift, suggested perturbation pressure minimum, and convergence-divergence signature). This highlights the question of whether these towers of enhanced $\zeta$ weakened solely as a result of the hurricane's landfall, changes to the inner core and MV maintenance processes after a prolonged $(10+\mathrm{h}) \mathrm{MV}$ episode, or perhaps most likely, some combination of both.

\section{Summary}

The kinematic structure of MVs in the inner core of Hurricane Ike (2008) is documented with nearly $10 \mathrm{~h}$ of ground-based dual-Doppler analysis. Initial results for a $160 \mathrm{~km} \times 160 \mathrm{~km} \times 10 \mathrm{~km}$ domain are obtained by applying the interpolated best track forward speed and direction for the motion of Ike as a uniform advection in 
the synthesis. Smaller domains (generally $30 \mathrm{~km} \times$ $30 \mathrm{~km} \times 10 \mathrm{~km})$ are then defined over potential MV features, and averaged wind retrievals from the initial domain are applied as a uniform advection in the synthesis over the smaller analysis grid. A total of 166 smaller domain analyses are completed, providing an unprecedented ground-based dual-Doppler view of MVs in the eyewall of a landfalling hurricane. Features containing a coherent local $\zeta$ maximum are deemed MVs. The most significant findings of this observational study are as follows:

- The largest $\zeta$ values are concentrated in the lowest $2 \mathrm{~km}$, consistent with previous studies.

- Arrangements of kinematic fields (Fig. 7) are pervasive for all MVs prior to multiple hours of land interaction, including a contiguous towerlike structure of enhanced $\zeta$ values (generally $\geq 4 \times 10^{-3} \mathrm{~s}^{-1}$ ) extending up to $8 \mathrm{~km}$.

- The MV updrafts preferentially occur radially outward from the local $\zeta$ maximum, appearing to rotate cyclonically around the $\zeta$ peak as the MVs occupy different eyewall regions.

- Within the first $2 \mathrm{~h}$ after landfall, the enhanced $\zeta$ towers become vertically confined to $4-6 \mathrm{~km}$. At later times, the structures continue to decay in height while the low-level $(1.5 \mathrm{~km}) \zeta$ maximum may or may not decrease (Fig. 8).

- Coinciding with the deterioration of the vertically coherent $\zeta$ structures, the characteristic kinematic field arrangements observed prior to landfall also decay.

Centers of MV $\zeta$ maxima are generally located near the leading (downstream) edge of the reflectivity arc signature $(\geq 30 \mathrm{~dB} Z)$, though in a few instances the $\zeta$ peak is more central to the reflectivity arc. The MV updrafts preferentially occur radially outward from the local $\zeta$ maximum, as characterized in Table 3 and Fig. 7 . Magnitudes of upward $w$ are largest on the upshear side of the storm (north-northeast). This is inconsistent with the conceptual model developed by Braun et al. (2006) and Braun and $\mathrm{Wu}$ (2007), though in their oceanic cases the additional complexities from landfall processes were not present. Based on values reported by Jorgensen et al. (1985) and Black et al. (1996), dual-Doppler-derived updraft magnitudes in Ike's MVs are within the upper $5 \%-10 \%$ of eyewall updrafts.

Horizontal wind convergence (divergence) tends to occur on the upstream (downstream) side of the MV $\zeta$ maximum. For MVs observed through 1-h postlandfall, pressure perturbation retrievals suggest a local MV pressure minimum, and a shift in the radial wind component generally coincides with the local $\zeta$ center.
Contours of radial wind and the pressure perturbation are nearly perpendicular for mature MVs, but as Ike progresses inland and the features weaken this arrangement is less striking, indicating decreased barotropic instability.

Vertically, the MV's arced reflectivity echo tops extend to about $8 \mathrm{~km}$, though a few are slightly taller. The entities contain peak $\zeta$ in the lowest $2 \mathrm{~km}$ and towerlike structures of enhanced $\zeta$ aloft. Over time, in some combination of the hurricane weakening as it moves inland and the demise of the MV episode, the $\zeta$ towers degrade in both magnitude and vertical extent (Fig. 8). Tower structures of enhanced $\zeta$ in the MVs suggest a rebuilding of a high $\zeta$ annulus, and resemble the more unstable regime 1 discussed by Kossin and Eastin (2001).

Unlike previous observational studies detailing MVs, to the authors' knowledge the present work is the first to provide a long-term (nearly $10 \mathrm{~h}$ ) ground-based dualDoppler analysis of inner core MVs and their attendant kinematic structure as they occupy almost every region of the eyewall (Table 3). We have shown that a welloriented network of ground-based Doppler radars can provide a continuous MV dataset, assuming that the observed TC develops an MV regime and passes within range of the network. Future work on the Ike event, including simulation studies, should address whether and to what extent the deterioration of the vorticity towers is due to land interaction and/or the dynamic progression of the MV episode.

Acknowledgments. This study would not have been possible without the dedicated field operations of the UAH Ike deployment crew: Dan Cecil, Patrick Gatlin, Cody Kirkpatrick, Kevin Knupp, Ken Leppert II, and Dustin Phillips. Comments from three helpful reviewers improved the quality of this paper. Support for this research was provided by NOAA Grant NA08OAR4600896.

\section{REFERENCES}

Aberson, S. D., M. T. Montgomery, M. Bell, and M. Black, 2006: Hurricane Isabel (2003): New insights into the physics of intense storms. Part II: Extreme localized wind. Bull. Amer. Meteor. Soc., 87, 1349-1354, doi:10.1175/BAMS-87-10-1349.

Armijo, L., 1969: A theory for the determination of wind and precipitation velocities with Doppler radars. J. Atmos. Sci., 26, 570573, doi:10.1175/1520-0469(1969)026<0570:ATFTDO >2.0.CO;2.

Asefi-Najafabady, S., K. Knupp, J. Mecikalski, R. Welch, and D. Phillips, 2010: Ground-based measurements and dualDoppler analysis of 3-D wind fields and atmospheric circulations induced by a meso- $\gamma$-scale inland lake. J. Geophys. Res., 115, D23117, doi:10.1029/2010JD014022.

Barnes, G. M., and M. D. Powell, 1995: Evolution of the inflow boundary layer of Hurricane Gilbert (1988). Mon. Wea. Rev., 
123, 2348-2368, doi:10.1175/1520-0493(1995)123<2348: EOTIBL $>2.0 . \mathrm{CO} ; 2$.

Bell, M. M., and M. T. Montgomery, 2008: Observed structure, evolution, and potential intensity of category-5 Hurricane Isabel (2003) from 12 to 14 September. Mon. Wea. Rev., 136, 2023-2046, doi:10.1175/2007MWR1858.1.

Berg, R., 2009: Tropical Cyclone Report: Hurricane Ike. National Hurricane Center Rep. AL092008, 55 pp. [Available online at http://www.nhc.noaa.gov/data/tcr/AL092008_Ike.pdf.]

Black, M. L., R. W. Burpee, and F. D. Marks, 1996: Vertical motion characteristics of tropical cyclones determined with airborne Doppler radial velocities. J. Atmos. Sci., 53, 1887-1909, doi:10.1175/1520-0469(1996)053<1887:VMCOTC > 2.0.CO;2.

Braun, S. A., and L. Wu, 2007: A numerical study of Hurricane Erin (2001). Part II: Shear and the organization of eyewall vertical motion. Mon. Wea. Rev., 135, 1179-1194, doi:10.1175/ MWR3336.1.

, M. T. Montgomery, and Z. Pu, 2006: High-resolution simulation of Hurricane Bonnie (1998). Part I: The organization of eyewall vertical motion. J. Atmos. Sci., 63, 19-42, doi:10.1175/ JAS3598.1.

Brown, D. P., J. L. Beven, J. L. Franklin, and E. S. Blake, 2010: Atlantic hurricane season of 2008. Mon. Wea. Rev., 138, 19752001, doi:10.1175/2009MWR3174.1.

Chong, M., and J. Testud, 1983: Three-dimensional wind field analysis from dual-doppler radar data. Part III: The boundary condition: An optimum determination based on a variational concept. J. Climate Appl. Meteor., 22, 1227-1241, doi:10.1175/ 1520-0450(1983)022<1227:TDWFAF $>2.0 . \mathrm{CO} ; 2$.

_, _, and F. Roux, 1983: Three-dimensional wind field analysis from dual-Doppler radar data. Part II: Minimizing the error due to temporal variation. J. Climate Appl. Meteor., 22, 1216-1226, doi:10.1175/1520-0450(1983)022<1216: TDWFAF $>2.0 . \mathrm{CO} ; 2$.

Clark, T. L., F. I. Harris, and C. G. Mohr, 1980: Errors in wind fields derived from multiple-Doppler radars: Random errors and temporal errors associated with advection and evolution. J. Appl. Meteor., 19, 1273-1284, doi:10.1175/ 1520-0450(1980)019<1273:EIWFDF>2.0.CO;2.

Cram, T. A., J. Persing, M. T. Montgomery, and S. A. Braun, 2007: A Lagrangian trajectory view on transport and mixing processes between the eye, eyewall, and environment using a high-resolution simulation of Hurricane Bonnie (1998). J. Atmos. Sci., 64, 1835-1856, doi:10.1175/JAS3921.1.

Doviak, R. J., and D. S. Zrnić, 1993: Doppler Radar and Weather Observations. Academic Press, 562 pp.

—, P. S. Ray, R. G. Strauch, and L. J. Miller, 1976: Error estimation in wind fields derived from dual-Doppler radar measurement. J. Appl. Meteor., 15, 868-878, doi:10.1175/ 1520-0450(1976)015<0868:EEIWFD>2.0.CO;2.

Eastin, M. D., P. G. Black, and W. M. Gray, 2005: Buoyancy of convective vertical motions in the inner core of intense hurricanes. Part II: Case studies. Mon. Wea. Rev., 133, 209-227, doi:10.1175/MWR-2849.1.

Emanuel, K. A., 1991: The theory of hurricanes. Annu. Rev. Fluid Mech., 23, 179-196, doi:10.1146/annurev.fl.23.010191.001143.

Gal-Chen, T., 1978: A method for the initialization of the anelastic equation: Implications for matching models with observations. Mon. Wea. Rev., 106, 587-606, doi:10.1175/ 1520-0493(1978)106<0587:AMFTIO > 2.0.CO;2.

Hane, C. E., R. B. Wilhelmson, and T. Gal-Chen, 1981: Retrieval of thermodynamic variables within deep convective clouds: Experiments in three dimensions. Mon.
Wea. Rev., 109, 564-576, doi:10.1175/1520-0493(1981)109<0564: ROTVWD $>2.0 . \mathrm{CO} ; 2$.

Hendricks, E. A., and W. H. Schubert, 2010: Adiabatic rearrangement of hollow PV towers. J. Adv. Model. Earth Syst., 2 (4), doi:10.3894/JAMES.2010.2.8.

,-- , R. K. Taft, H. Wang, and J. P. Kossin, 2009: Life cycles of hurricane-like vorticity rings. J. Atmos. Sci., 66, 705-722, doi:10.1175/2008JAS2820.1.

- B. D. McNoldy, and W. H. Schubert, 2012: Observed innercore structural variability in Hurricane Dolly (2008). Mon. Wea. Rev., 140, 4066-4077, doi:10.1175/MWR-D-12-00018.1.

—, W. H. Schubert, Y.-H. Chen, H.-C. Kuo, and M. S. Peng, 2014: Hurricane eyewall evolution in a forced shallow-water model J. Atmos. Sci., 71, 1623-1643, doi:10.1175/JAS-D-13-0303.1.

Jorgensen, D. P., E. J. Zipser, and M. A. LeMone, 1985: Vertical motions in intense hurricanes. J. Atmos. Sci., 42, 839-856, doi:10.1175/1520-0469(1985)042<0839:VMIIH>2.0.CO;2.

Kossin, J. P., and M. D. Eastin, 2001: Two distinct regimes in the kinematic and thermodynamic structure of the hurricane eye and eyewall. J. Atmos. Sci., 58, 1079-1090, doi:10.1175/ 1520-0469(2001)058<1079:TDRITK > 2.0.CO;2.

— terns, and rapid pressure falls in hurricane-like vortices. J. Atmos. Sci., 58, 2196-2209, doi:10.1175/1520-0469(2001)058<2196: MPFPAR $>2.0 . \mathrm{CO} ; 2$.

$\longrightarrow$, and —, 2004: Mesovortices in Hurricane Isabel. Bull. Amer. Meteor. Soc., 85, 151-153, doi:10.1175/BAMS-85-2-151.

Lewis, B., and H. Hawkins, 1982: Polygonal eye walls and rainbands in hurricanes. Bull. Amer. Meteor. Soc., 63, 1294-1301, doi:10.1175/1520-0477(1982)063<1294:PEWARI>2.0.CO;2.

Li, X., J. A. Zhang, X. Yang, W. G. Pichel, M. DeMaria, D. Long, and Z. Li, 2013: Tropical cyclone morphology from spaceborne synthetic aperture radar. Bull. Amer. Meteor. Soc., 94, 215-230, doi:10.1175/BAMS-D-11-00211.1

Marks, F. D., and R. A. Houze, 1984: Airborne Doppler radar observations in Hurricane Debby. Bull. Amer. Meteor. Soc., 65, 569582, doi:10.1175/1520-0477(1984)065<0569:ADROIH > 2.0.CO;2. , P. G. Black, M. T. Montgomery, and R. W. Burpee, 2008: Structure of the eye and eyewall of Hurricane Hugo (1989). Mon. Wea. Rev., 136, 1237-1259, doi:10.1175/2007MWR2073.1.

Martinez, Y. H., G. Brunet, and M. K. Yau, 2010: On the dynamics of two-dimensional hurricane-like vortex symmetrization. J. Atmos. Sci., 67, 3559-3580, doi:10.1175/2010JAS3499.1.

Matejka, T., and D. L. Bartels, 1998: The accuracy of vertical air velocities from Doppler radar data. Mon. Wea. Rev., 126, 92-117, doi:10.1175/1520-0493(1998)126<0092:TAOVAV>2.0.CO;2.

Menelaou, K., M. K. Yau, and Y. Martinez, 2013: On the origin and impact of a polygonal eyewall in the rapid intensification of Hurricane Wilma (2005). J. Atmos. Sci., 70, 3839-3858, doi:10.1175/ JAS-D-13-091.1.

Miller, L. J., and R. G. Strauch, 1974: A dual Doppler radar method for the determination of wind velocities within precipitating weather systems. Remote Sens. Environ., 3, 219-235, doi:10.1016/ 0034-4257(74)90044-3.

Mohr, C. G., L. J. Miller, R. L. Vaughn, and H. W. Frank, 1986: The merger of mesoscale datasets into a common Cartesian format for efficient and systematic analyses. J. Atmos. Oceanic Technol., 3, 143-161, doi:10.1175/1520-0426(1986)003<0143: TMOMDI $>2.0 . \mathrm{CO} ; 2$.

Molinari, J., P. Dodge, D. Vollaro, K. Corbosiero, and F. Marks, 2006: Mesoscale aspects of the downshear reformation of a tropical cyclone. J. Atmos. Sci., 63, 341-354, doi:10.1175/ JAS3591.1. 
Montgomery, M. T., V. A. Vladimirov, and P. V. Denissenko, 2002: An experimental study on hurricane mesovortices. J. Fluid Mech., 471, 1-32, doi:10.1017/S0022112002001647.

_- M. M. Bell, S. D. Aberson, and M. Black, 2006: Hurricane Isabel (2003): New insights into the physics of intense storms. Part I: Mean vortex structure and maximum intensity estimates. Bull. Amer. Meteor. Soc., 87, 1335-1347, doi:10.1175/ BAMS-87-10-1335.

Murphy, T. A., and K. R. Knupp, 2013: An analysis of cold season supercell storms using the synthetic dual-Doppler technique. Mon. Wea. Rev., 141, 602-624, doi:10.1175/MWR-D-12-00035.1.

Naylor, J., and D. A. Schecter, 2014: Evaluation of the impact of moist convection on the development of asymmetric inner core instabilities in simulated tropical cyclones. J. Adv. Model. Earth Syst., 6, 1027-1048, doi:10.1002/2014MS000366.

Nguyen, L. T., and J. Molinari, 2015: Simulation of the downshear reformation of a tropical cyclone. J. Atmos. Sci., 72, 45294551, doi:10.1175/JAS-D-15-0036.1.

Persing, J., and M. T. Montgomery, 2003: Hurricane superintensity. J. Atmos. Sci., 60, 2349-2371, doi:10.1175/ 1520-0469(2003)060<2349:HS > 2.0.CO;2.

Ray, P. S., M. Gilet, and K. W. Johnson, 1980: The multiple Doppler radar workshop, November 1979. Part IV: Motion field synthesis and radar placement. Bull. Amer. Meteor. Soc., 61, 1184-1189, doi:10.1175/1520-0477(1980)061<1184: PIMFSA $>2.0 . \mathrm{CO} ; 2$.

Reasor, P. D., M. T. Montgomery, F. D. Marks, and J. F. Gamache, 2000: Low-wavenumber structure and evolution of the hurricane inner core observed by airborne dual-Doppler radar. Mon. Wea. Rev., 128, 1653-1680, doi:10.1175/1520-0493(2000)128<1653: LWSAEO $>2.0 . \mathrm{CO} ; 2$.

— M. D. Eastin, and J. F. Gamache, 2009: Rapidly intensifying Hurricane Guillermo (1997). Part I: Low-wavenumber structure and evolution. Mon. Wea. Rev., 137, 603-631, doi:10.1175/ 2008MWR2487.1.

Roux, F., 1985: Retrieval of thermodynamic fields from multipleDoppler radar data using the equations of motion and the thermodynamic equation. Mon. Wea. Rev., 113, 2142-2157, doi:10.1175/1520-0493(1985)113<2142:ROTFFM > 2.0.CO;2.

Rozoff, C. M., J. P. Kossin, W. H. Schubert, and P. J. Mulero, 2009: Internal control of hurricane intensity variability: The dual nature of potential vorticity mixing. J. Atmos. Sci., 66, 133147, doi:10.1175/2008JAS2717.1.

Schubert, W. H., M. T. Montgomery, R. K. Taft, T. A. Guinn, S. R. Fulton, J. P. Kossin, and J. P. Edwards, 1999: Polygonal eyewalls, asymmetric eye contraction, and potential vorticity mixing in hurricanes. J. Atmos. Sci., 56, 1197-1223, doi:10.1175/ 1520-0469(1999)056<1197:PEAECA > 2.0.CO;2.

Sitkowski, M., and G. M. Barnes, 2009: Low-level thermodynamic, kinematic, and reflectivity fields of Hurricane Guillermo (1997) during rapid intensification. Mon. Wea. Rev., 137, 645663, doi:10.1175/2008MWR2531.1.

Terwey, W. D., and M. T. Montgomery, 2002: Wavenumber-2 and wavenumber- $m$ vortex Rossby wave instabilities in a generalized three-region model. J. Atmos. Sci., 59, 2421-2427, doi:10.1175/1520-0469(2002)059<2421:WAWMVR>2.0.CO;2.

Testud, J., and M. Chong, 1983: Three-dimensional wind field analysis from dual-Doppler radar data. Part I: Filtering, interpolating, and differentiating the raw data. J. Climate Appl. Meteor., 22, 1204-1215, doi:10.1175/1520-0450(1983)022<1204: TDWFAF $>2.0 . \mathrm{CO} ; 2$.

Willoughby, H., and P. Black, 1996: Hurricane Andrew in Florida: Dynamics of a disaster. Bull. Amer. Meteor. Soc., 77, 543-549, doi:10.1175/1520-0477(1996)077<0543:HAIFDO>2.0.CO;2.

Wroe, D. R., and G. M. Barnes, 2003: Inflow layer energetics of Hurricane Bonnie (1998) near landfall. Mon. Wea. Rev., 131, 1600-1612, doi:10.1175//2547.1.

Wu, C.-C., H.-J. Cheng, Y. Wang, and K.-H. Chou, 2009: A numerical investigation of the eyewall evolution in a landfalling typhoon. Mon. Wea. Rev., 137, 21-40, doi:10.1175/2008MWR2516.1.

—, S.-N. Wu, H.-H. Wei, and S. F. Abarca, 2016: The role of convective heating in tropical cyclone eyewall ring evolution. J. Atmos. Sci., 73, 319-330, doi:10.1175/JAS-D-15-0085.1. 